\title{
Markov Neighborhood Regression for High-Dimensional Inference
}

\author{
Faming Liang, Jingnan Xue, Bochao Jia*
}

October 20, 2020

\begin{abstract}
This paper proposes an innovative method for constructing confidence intervals and assessing $p$-values in statistical inference for high-dimensional linear models. The proposed method has successfully broken the high-dimensional inference problem into a series of low-dimensional inference problems: For each regression coefficient $\beta_{i}$, the confidence interval and $p$-value are computed by regressing on a subset of variables selected according to the conditional independence relations between the corresponding variable $X_{i}$ and other variables. Since the subset of variables forms a Markov neighborhood of $X_{i}$ in the Markov network formed by all the variables $X_{1}, X_{2}, \ldots, X_{p}$, the proposed method is coined as Markov neighborhood regression. The proposed method is tested on high-dimensional linear, logistic and Cox regression. The numerical results indicate that the proposed method significantly outperforms the existing ones. Based on the Markov neighborhood regression, a method of learning causal structures for high-dimensional linear models is proposed and applied to identification of drug sensitive genes and cancer driver genes. The idea of using conditional independence relations for dimension reduction is general and potentially can be extended to other high-dimensional or big data problems as well.
\end{abstract}

Keywords: Confidence Interval; Causal Structure Discovery; Gaussian Graphical Model; p-value

\section{Introduction}

During the past two decades, the dramatic improvement in data collection and acquisition technologies has enabled scientists to collect a great amount of high-dimensional data, for which the dimension $p$ can be much larger than the sample size $n$ (a.k.a. small- $n$-large- $p$ ). The current research on high-dimensional data mainly focuses on variable selection and graphical modeling. The former aims to find a consistent estimate for high-dimensional regression under a sparsity constraint. The existing methods include Lasso (Tibshirani, 1996), SCAD (Fan and Li, 2001), MCP (Zhang, 2010), and rLasso (Song and Liang, 2015a), among others. The latter aims to learn conditional independence relationships for a large set of variables. The existing methods include graphical Lasso (Yuan and Lin, 2007; Friedman et al., 2008), nodewise regression (Meinshausen and Bühlmann, 2006), and $\psi$-learning (Liang et al., 2015), among others.

Recently many researchers turn their attention to statistical inference for high-dimensional data, aiming to quantify uncertainty of high-dimensional regression, e.g., constructing confidence intervals and assessing

${ }^{*}$ Liang is Professor, Department of Statistics, Purdue University, West Lafayette, IN 47906, email: fmliang@purdue.edu. Xue is Data Scientist at Houzz, Palo Alto, California. Jia is Research Scientist, Eli Lilly and Company, Lilly Corporate Center, Indianapolis, IN 46285. 
$p$-values for the regression coefficients. The Bayesian methods (Liang et al., 2013; Song and Liang, 2015b) can potentially be used for this purpose, but are time-consuming. The existing frequentist methods include desparsified Lasso, multi sample-splitting, and ridge projection, among others. Refer to Dezeure et al. (2015) for a comprehensive overview.

The desparsified Lasso method was proposed in van de Geer et al. (2014), which is also essentially the same as the one developed in Zhang and Zhang (2014) and Javanmard and Montanari (2014). For the highdimensional linear regression

$$
\boldsymbol{Y}=\boldsymbol{X} \boldsymbol{\beta}+\boldsymbol{\epsilon}
$$

where $\boldsymbol{\epsilon}$ are zero-mean Gaussian random errors, desparsified Lasso defines a bias-corrected estimator

$$
\hat{\boldsymbol{\beta}}_{b c}=\hat{\boldsymbol{\beta}}_{\text {Lasso }}+\hat{\Theta} \boldsymbol{X}^{T}\left(\boldsymbol{y}-\boldsymbol{X} \hat{\boldsymbol{\beta}}_{\text {Lasso }}\right) / n,
$$

where $\hat{\boldsymbol{\beta}}_{\text {Lasso }}$ is the original Lasso estimator, and $\hat{\Theta}$ is an approximator to the inverse of $\hat{\boldsymbol{\Sigma}}=\boldsymbol{X}^{T} \boldsymbol{X} / n$. From (1), one can obtain

$$
\sqrt{n}\left(\hat{\boldsymbol{\beta}}_{b c}-\boldsymbol{\beta}\right)=\hat{\Theta} \boldsymbol{X}^{T} \boldsymbol{\epsilon} / \sqrt{n}+\sqrt{n}\left(I_{p}-\hat{\Theta} \hat{\boldsymbol{\Sigma}}\right)\left(\hat{\boldsymbol{\beta}}_{\text {Lasso }}-\boldsymbol{\beta}\right):=\hat{\Theta} \boldsymbol{X}^{T} \boldsymbol{\epsilon} / \sqrt{n}+\Delta_{n},
$$

where $I_{p}$ denotes the $p \times p$ identity matrix, and $\Delta_{n}$ is the error term. With an appropriate estimator $\hat{\Theta}$, e.g., the one obtained by nodewise regression (Meinshausen and Bühlmann, 2006), it can be shown that $\left\|\Delta_{n}\right\|_{\infty}=o_{p}(1)$ and thus $\sqrt{n}\left(\hat{\boldsymbol{\beta}}_{b c}-\boldsymbol{\beta}\right)$ shares the same asymptotic distribution with $\hat{\Theta} \boldsymbol{X}^{T} \boldsymbol{\epsilon} / \sqrt{n}$. Further, to calculate confidence intervals for $\boldsymbol{\beta}$, one needs to approximate the distribution of $\hat{\Theta} \boldsymbol{X}^{T} \boldsymbol{\epsilon} / \sqrt{n}$. For example, Javanmard and Montanari (2014) approximated it by $N\left(0, \hat{\sigma}^{2} \hat{\Theta} \hat{\Sigma} \hat{\Theta}^{T}\right)$, where $\hat{\sigma}^{2}$ is a consistent estimator of $\sigma^{2}$; and Zhang and Cheng (2017) approximated it using multiplier bootstrap.

The multi sample-splitting method was proposed and analyzed in Meinshausen et al. (2009), which works in the following procedure: Splitting the samples into two subsets equally, using the first half of samples for variable selection and using the second half of samples for calculating $p$-values based on the selected variables; repeating this process for many times; and aggregating the $p$-values for statistical inference. The confidence intervals can be constructed based on their duality with $p$-values. The idea about sample-splitting and subsequent statistical inference has also been implicitly contained in Wasserman and Roeder (2009). The multi sample-splitting method is very general and can be applied to many different types of models. The ridge projection method was studied in Bühlmann (2013), which can be viewed as a direct extension of the low-dimensional ridge regression to the high-dimensional case. The bias of the ridge estimator has been assessed and corrected, the distribution of the ridge estimator has been derived and approximated, and thus the method can be used for statistical inference.

The other methods include residual-type bootstrapping (Chatteriee and Lahiri, 2013; Liu and Yu, 2013), covariance test (Lockhart et al., 2014), and group-bound (Meinshausen, 2015). A problem with the residualtype bootstrapping method is the super-efficiency phenomenon; that is, a confidence interval of a zero regression coefficient might be the singleton $\{0\}$. The covariance test method relies on the solution path of the Lasso and is much related to the post-selection inference methods (Berk et al., 2013; Lee et al., 2016b; Tibshirani et al., 2016; Fithian et al., 2014). The group-bound method provides a good treatment for highly correlated variables, but has often a weak power in detecting the effect of individual variables. Recently, some methods based on the idea of estimating a low-dimensional component of a high-dimensional model have also been proposed, see e.g. Belloni et al. (2015) and Yang (2017). 
This paper proposes an innovative method, the so-called Markov neighborhood regression (MNR), for high-dimensional inference. By making use of conditional independence relations among different explanatory variables, it successfully breaks the high-dimensional inference problem into a series of low-dimensional inference problems. The proposed method is fundamentally different from the existing ones, such as desparsified-Lasso, ridge projection, and multi sample-splitting, which strive to work on the problem in its original scale. The proposed method has been tested on high-dimensional linear, logistic and Cox regression. The numerical results indicate that the proposed method significantly outperforms the existing ones, while having competitive computational efficiency. Based on the concept of MNR, this paper also proposes a new method for learning the causal structure of high-dimensional regression and applies it to identification of drug sensitive genes and cancer driver genes. The idea of using conditional independence for dimension reduction is general and can be applied to many other high-dimensional or big data problems as well.

The remaining part of this paper is organized as follows. Section 2 describes the MNR method and establishes its validity. Section 3 presents numerical results on simulated data along with comparisons with some existing methods. Section 4 proposes a method for learning the causal structures of high-dimensional regression. Section 5 presents some real data examples. Section 6 concludes the paper with a brief discussion.

\section{Markov Neighborhood Regression}

\subsection{Linear Regression}

Suppose that a set of $n$ independent samples $D_{n}=\left\{\left(Y^{(i)}, \boldsymbol{X}^{(i)}\right)_{i=1}^{n}\right\}$ have been collected from the linear regression with a random design:

$$
Y=\beta_{0}+X_{1} \beta_{1}+\ldots+X_{p} \beta_{p}+\epsilon,
$$

where $\epsilon$ follows the normal distribution $N\left(0, \sigma^{2}\right)$, and the explanatory variables, or known as features, $\boldsymbol{X}=$ $\left(X_{1}, \ldots, X_{p}\right)$ follows a multivariate normal distribution $N_{p}(\mathbf{0}, \Sigma)$. In what follows, we will call $\left\{X_{i}: \beta_{i} \neq 0, i=\right.$ $1,2, \ldots, p\}$ and $\left\{X_{i}: \beta_{i}=0, i=1,2, \ldots, p\right\}$ the true and false features, respectively. Without loss of generality, we assume that the features have been standardized such that $E\left(X_{j}\right)=0$ and $\operatorname{Var}\left(X_{j}\right)=1$ for $j=1, \ldots, p$. Further, we represent $\boldsymbol{X}$ by an undirected graph $\boldsymbol{G}=(\boldsymbol{V}, \boldsymbol{E})$, where $\boldsymbol{V}=\{1,2, \ldots, p\}$ denotes the set of $p$ vertices, $\boldsymbol{E}=\left(e_{i j}\right)$ denotes the adjacency matrix, $e_{i j}=1$ if the $(i, j)$ th entry of the precision matrix $\Theta=\Sigma^{-1}$ is nonzero and 0 otherwise. We use $\boldsymbol{X}_{\boldsymbol{A}}=\left\{X_{k}: k \in \boldsymbol{A}\right\}$ to denote a set of features indexed by $\boldsymbol{A} \subset \boldsymbol{V}$, and use $P_{\boldsymbol{V}}$ to denote the joint probability distribution of $\boldsymbol{X}_{\boldsymbol{V}}$. For a triplet $\boldsymbol{I}, \boldsymbol{J}, \boldsymbol{U} \subset \boldsymbol{V}$, we use $\boldsymbol{X}_{\boldsymbol{I}} \perp \boldsymbol{X}_{\boldsymbol{J}} \mid \boldsymbol{X}_{\boldsymbol{U}}$ to denote that $\boldsymbol{X}_{\boldsymbol{I}}$ is conditionally independent of $\boldsymbol{X}_{\boldsymbol{J}}$ given $\boldsymbol{X}_{\boldsymbol{U}}$. A path of length $l>0$ from a vertex $v_{0}$ to another vertex $v_{l}$ is a sequence $v_{0}, v_{1}, \ldots, v_{l}$ of distinct vertices such that $e_{v_{k-1}, v_{k}}=1$ for $k=1,2, \ldots, l$. The subset $\boldsymbol{U} \subset \boldsymbol{V}$ is said to separate $\boldsymbol{I} \subset \boldsymbol{V}$ from $\boldsymbol{J} \subset \boldsymbol{V}$ if for every $i \in \boldsymbol{I}$ and $j \in \boldsymbol{J}$, all paths from $i$ to $j$ have at least one vertex in $\boldsymbol{U} . P_{\boldsymbol{V}}$ is said to satisfy the Markov property with respect to $\boldsymbol{G}$ if for every triple of disjoint sets $\boldsymbol{I}, \boldsymbol{J}, \boldsymbol{U} \subset \boldsymbol{V}$, it holds that $X_{\boldsymbol{I}} \perp X_{\boldsymbol{J}} \mid X_{\boldsymbol{U}}$ whenever $\boldsymbol{U}$ separates $\boldsymbol{I}$ and $\boldsymbol{J}$ in $\boldsymbol{G}$. Let $\xi_{j}=\left\{k: e_{j k}=1\right\}$ denote the neighboring set of $X_{j}$ in $G$. Following from the Markov property of the Gaussian graphical model (GGM), we have $X_{j} \perp X_{i} \mid \boldsymbol{X}_{\xi_{j}}$ for any $i \in \boldsymbol{V} \backslash \xi_{j}$, as $\xi_{j}$ forms a separator between $X_{i}$ and $X_{j}$. For convenience, we call $\xi_{j}$ the minimum Markov neighborhood of $X_{j}$ in $\boldsymbol{G}$, and call any superset $\boldsymbol{s}_{j} \supset \xi_{j}$ a Markov neighborhood of $X_{j}$ in $\boldsymbol{G}$. The minimum Markov neighborhood is also termed as Markov blanket in Bayesian networks or general Markov networks.

To motivate the proposed method, we first look at a simple mathematical fact based on the well known 
property of Gaussian graphical models (see e.g. Lauritzen (1996)):

$$
X_{i} \perp X_{j} \mid X_{\boldsymbol{V} \backslash\{i, j\}} \Longleftrightarrow \theta_{i j}=0,
$$

where $\theta_{i j}$ denotes the $(i, j)$-th entry of $\Theta$. Without loss of generality, we let $\boldsymbol{S}_{1}=\{2, \ldots, d\}$ denote a Markov neighborhood of $X_{1}$, let $\Sigma_{d}$ denote the covariance matrix of $\left\{X_{1}\right\} \cup \boldsymbol{X}_{\boldsymbol{S}_{1}}$, and partition $\Theta$ as

$$
\Theta=\left[\begin{array}{cc}
\Theta_{d} & \Theta_{d, p-d} \\
\Theta_{p-d, d} & \Theta_{p-d}
\end{array}\right],
$$

where the first row of $\Theta_{d, p-d}$ and the first column of $\Theta_{p-d, d}$ are exactly zero, as $X_{1} \perp \boldsymbol{X}_{\boldsymbol{V} \backslash\left(\{1\} \cup \boldsymbol{S}_{1}\right)} \mid \boldsymbol{X}_{\boldsymbol{S}_{1}}$ holds. Inverting $\Theta$, we have $\Sigma_{d}=\left(\Theta_{d}-\Theta_{d, p-d} \Theta_{p-d}^{-1} \Theta_{p-d, d}\right)^{-1}$, which is equal to the top $d \times d$-submatrix of $\Theta^{-1}$. Therefore,

$$
\Sigma_{d}^{-1}=\Theta_{d}-\Theta_{d, p-d} \Theta_{p-d}^{-1} \Theta_{p-d, d}
$$

Since the first row of $\Theta_{d, p-d}$ and the first column of $\Theta_{p-d, d}$ are exactly zero, the $(1,1)$ th element of $\Theta_{d, p-d} \Theta_{p-d}^{-1} \Theta_{p-d, d}$ is exactly zero. Therefore, the $(1,1)$-th entry of $\Theta_{d}$ (and $\Theta$ ) equals to the $(1,1)$-th entry of $\Sigma_{d}^{-1}$. This suggests that if $\left\{X_{1}\right\} \cup \boldsymbol{X}_{\boldsymbol{S}_{1}} \supset \boldsymbol{X}_{\boldsymbol{S}_{*}}$ holds and $n$ is sufficiently large, where $\boldsymbol{S}_{*}$ denote the index set of true features of the model (3), then the statistical inference for $\beta_{1}$ can be made based on the subset regression:

$$
Y=\beta_{0}^{\prime}+X_{1} \beta_{1}+X_{2} \beta_{2}^{\prime}+\ldots+X_{d} \beta_{d}^{\prime}+\epsilon
$$

where the prime on $\beta_{k}$ 's for $k \neq 1$ indicates that those regression coefficients might be modified by the subset regression. Since $\boldsymbol{S}_{1}$ forms a Markov neighborhood of $X_{1}$ in the Markov network formed by all features, we call (6) a Markov neighborhood regression, which breaks the high-dimensional inference problem into a series of low-dimensional inference problems. Based on this mathematical fact, we propose the following algorithm:

Algorithm 1. (Markov Neighborhood Regression)

(a) (Variable selection) Conduct variable selection for the model (3) to get a consistent estimate of $\boldsymbol{S}_{*}$. Denote the estimate by $\hat{\boldsymbol{S}}_{*}$.

(b) (Markov blanket estimation) Construct a GGM for $\boldsymbol{X}$ and obtain a consistent estimate of the Markov blanket for each variable. Denote the estimates by $\hat{\xi}_{j}$ for $j=1,2, \ldots, p$.

(c) (Subset regression) For each variable $X_{j}, j=1, \ldots, p$, let $D_{j}=\{j\} \cup \hat{\xi}_{j} \cup \hat{\boldsymbol{S}}_{*}$ and run an Ordinary Least Square (OLS) regression with the features given by $\boldsymbol{X}_{D_{j}}$, i.e.,

$$
Y=\beta_{0}+\boldsymbol{X}_{D_{j}} \boldsymbol{\beta}_{D_{j}}+\epsilon
$$

where $\epsilon \sim N\left(0, \sigma^{2} I_{n}\right)$ and $I_{n}$ is an $n \times n$-identity matrix. Conduct inference for $\beta_{j}$, including the estimate, confidence interval and p-value, based on the output of (7).

The Markov neighborhood corresponding to the subset regression (6) is $\{2,3, \ldots, d\} \supseteq \hat{\xi}_{1} \cup \hat{\boldsymbol{S}}_{*}$. In general, $\hat{\xi}_{1} \cup \hat{\boldsymbol{S}}_{*}$ can be any a subset of $\{1,2, \ldots, p\}$ depending on the ordering of features in (3). Algorithm 1 can be implemented in many different ways. For example, a variety of high-dimensional variable selection algorithms can be used for step (a), e.g., Lasso (Tibshirani, 1996), SCAD (Fan and Li, 2001), MCP (Zhang, 2010) and 
rLasso (Song and Liang, 2015a), which are all able to produce a consistent estimate for $\boldsymbol{S}_{*}$ under appropriate conditions. Similarly, a variety of graphical model learning algorithms can be used for step (b), e.g., graphical Lasso (Yuan and Lin, 2007; Friedman et al., 2008), nodewise regression (Meinshausen and Bühlmann, 2006), and $\psi$-learning (Liang et al., 2015), which all produce a consistent estimate for the underlying GGM under appropriate conditions. To justify Algorithm 1, we introduce the following lemma, which can be proved based on the basic theory of high-dimensional linear regression and the mathematical relation shown around equations (44) and (5). Refer to the Supplementary Material for the detail of the proof.

Lemma 1. Let $\hat{\xi}_{j} \supseteq \xi_{j}$ denote any Markov neighborhood of feature $x_{j}$, let $\hat{\boldsymbol{S}}_{*} \supseteq \boldsymbol{S}_{*}$ denote any reduced feature space, and let $D_{j}=\{j\} \cup \hat{\xi}_{j} \cup \hat{\boldsymbol{S}}_{*}$. Consider the subset regression (7). Let $\hat{\boldsymbol{\beta}}_{D_{j}}$ denote the OLS estimator of $\boldsymbol{\beta}_{D_{j}}$ from the subset regression, and let $\hat{\beta}_{j}$ denote the element of $\hat{\boldsymbol{\beta}}_{D_{j}}$ corresponding to the variable $X_{j}$. If $\left|D_{j}\right|=o\left(n^{1 / 2}\right)$, as $n \rightarrow \infty$, the following results hold:

(i) $\sqrt{n}\left(\hat{\beta}_{j}-\beta_{j}\right) \sim N\left(0, \sigma^{2} \theta_{j j}\right)$, where $\theta_{j j}$ is the $(j, j)$-th entry of the precision matrix $\Theta$.

(ii) $\sqrt{n} \frac{\hat{\beta}_{j}-\beta_{j}}{\sqrt{\hat{\sigma}_{n}^{2}} \hat{\theta}_{j j}} \sim N(0,1)$, where $\hat{\sigma}_{n}^{2}=\left(\boldsymbol{y}-\boldsymbol{x}_{D_{j}} \hat{\boldsymbol{\beta}}_{D_{j}}\right)^{T}\left(\boldsymbol{y}-\boldsymbol{x}_{D_{j}} \hat{\boldsymbol{\beta}}_{D_{j}}\right) /(n-d-1), \hat{\theta}_{j j}$ is the $(j, j)$-th entry of the matrix $\left[\frac{1}{n} \sum_{i=1}^{n} \boldsymbol{x}_{D_{j}}^{(i)}\left(\boldsymbol{x}_{D_{j}}^{(i)}\right)^{T}\right]^{-1}$, and $\boldsymbol{x}_{D_{j}}^{(i)}$ denotes the $i$-th row of $\boldsymbol{X}_{D_{j}}$.

Remark 1. For the case that $n$ is finite, we have $\left(n-\left|D_{j}\right|-1\right) \hat{\sigma}_{n}^{2} / \sigma^{2} \sim \chi^{2}\left(n-\left|D_{j}\right|-1\right)$, independent of $\hat{\boldsymbol{\beta}}_{D_{j}}$, by the standard theory of OLS estimation. Therefore, we can use $t\left(n-\left|D_{j}\right|-1\right)$ to approximate the distribution of $\sqrt{n} \frac{\hat{\beta}_{j}-\beta_{j}}{\sqrt{\hat{\sigma}_{n}^{2} \hat{\theta}_{j j}}}$; that is, the estimate, p-value and confidence interval of $\beta_{j}$ can be calculated from (7) as in conventional low-dimensional multiple linear regression.

Lemma 1 implies that Algorithm 1 will be valid as long as the following conditions hold:

$$
\begin{aligned}
\hat{\xi}_{j} & \supseteq \xi_{j}, \forall j \in\{1,2, \ldots, p\}, \\
\hat{\boldsymbol{S}}_{*} & \supseteq \boldsymbol{S}_{*}, \\
\left|D_{j}\right| & =o(\sqrt{n}) .
\end{aligned}
$$

Condition (9) is the so-called screening property, which is known to be satisfied by many high-dimensional variable selection algorithms, such as SCAD (Fan and Li, 2001), MCP (Zhang, 2010) and adaptive Lasso (Zou, 2006). Lasso also satisfies this condition if the design matrix satisfies the compatibility condition (van de Geer and Bühlmann, 2009), $\left|\boldsymbol{S}_{*}\right|=o(n / \log (p))$, and the beta-min condition holds. See Dezeure et al. (2015) for more discussions on this issue. Given the sure screening property of the above variable selection procedure, if the nodewise regression algorithm (Meinshausen and Bühlmann, 2006) is applied to learn the GGM in step (b) of Algorithm 1, then the condition (8) can be satisfied. In fact, as along as the GGM construction algorithm is consistent, the condition (8) will be asymptotically satisfied. Further, the condition (10) can be easily satisfied by a slight twist of the sparsity conditions used in the variable selection and GGM estimation algorithms.

As an example, we give in the Appendix a set of technical conditions (A0)-(A9) under which the conditions (8) - (10) can be asymptotically satisfied, provided that the SCAD algorithm is used for variable selection and the $\psi$-learning algorithm is used for GGM estimation. Based on these technical conditions, the validity of Algorithm 1 is justified in Theorem 1, whose proof is straightforward based on Slutsky's theorem and some 
existing theoretical results. Refer to the Supplementary Material for the detail. If different algorithms are used in Algorithm 1, then the conditions used in Theorem 1 should be changed accordingly. We note that many conditions we imposed in proving the theorem are purely technical and only serve to provide theoretical understanding of the proposed method. We have no intent to make the conditions the weakest possible.

Theorem 1. (Validity of Algorithm 1) If the conditions (A0)-(A9) hold, the SCAD algorithm is used for variable selection in step (a), and the $\psi$-learning algorithm is used for GGM construction in step (b), then for each $j \in\left\{1,2, \ldots, p_{n}\right\}$, we have $\sqrt{n} \frac{\hat{\beta}_{j}-\beta_{j}}{\sqrt{\hat{\sigma}_{n}^{2} \hat{\theta}_{j j}}} \sim N(0,1)$ as $n \rightarrow \infty$, where $\hat{\beta}_{j}$ denotes the estimate of $\beta_{j}$ obtained from the subset regression, $\hat{\sigma}_{n}^{2}=\left(\boldsymbol{y}-\boldsymbol{x}_{D_{j}} \hat{\boldsymbol{\beta}}_{D_{j}}\right)^{T}\left(\boldsymbol{y}-\boldsymbol{x}_{D_{j}} \hat{\boldsymbol{\beta}}_{D_{j}}\right) /(n-d-1), \hat{\theta}_{j j}$ is the $(j, j)$-th entry of the matrix $\left[\frac{1}{n} \sum_{i=1}^{n} \boldsymbol{x}_{D_{j}}^{(i)}\left(\boldsymbol{x}_{D_{j}}^{(i)}\right)^{T}\right]^{-1}$, and $\boldsymbol{x}_{D_{j}}^{(i)}$ denotes the $i$-th row of $\boldsymbol{X}_{D_{j}}$.

Remark 2. Following Remark 1, we can conduct inference for $\beta_{j}$ based on the output of the subset regression (7) as in conventional low-dimensional multiple linear regression.

As implied by Theorem 1, variable selection for regression (3) can be converted as a multiple hypothesis testing problem for simultaneously testing the hypotheses

$$
H_{0, j}: \beta_{j}=0 \Longleftrightarrow H_{1, j}: \beta_{j} \neq 0, \quad j=1,2, \ldots, p
$$

based on the $p$-values obtained from the subset regressions. The consistency of this test-based method follows from Theorem 2 of Liang et al. (2015) as discussed in Section 4.1. Compared to the regularization methods, e.g. Lasso, MCP, SCAD and adaptive Lasso Zou (2006), a significant advantage of this method is that it controls the false discovery rate of selected features in an explicit way. In addition, since the screening property generally holds for these regularization methods, see Dezeure et al. (2015) for discussions on this issue, the new method might result in a lower false discovery rate as shown in Table 2. On the other hand, since the $p$-value measures the contribution of a feature to the regression conditioned on all other $p-1$ features, MNR might not work well when strong collinearity exists among certain true and false features. This case has been excluded by Conditions A2 and A4, where the fixed upper bounds on correlations and $\psi$-partial correlations place some additional restrictions on the design.

The essential conditions required by MNR are only sparsity; that is, the true regression model is sparse and the conditional independence structure among the features is sparse such that (8)-(10) hold when appropriate algorithms are applied. Similar conditions have been assumed by some existing methods. For example, desparsified-Lasso requires the true model to be of size $o(\sqrt{n} / \log p)$ (see e.g., Fact 2 of Dezeure et al. (2015)), which is a little more restrictive than $o\left(n^{1 / 2}\right)$ required by MNR; desparsified-Lasso also requires the precision matrix $\Theta$ to be row-sparse at a level of $o(n / \log p)$, which is comparable with the Markov blanket size $o(\sqrt{n})$ required by $\mathrm{MNR}$ when $\log (p)=n^{\delta}$ for some $\delta \approx 1 / 2$. The multi sample-splitting and the ridge projection methods require the screening property (9) only, which seems weaker than the conditions required by MNR and desparsified-Lasso. However, as shown later by numerical examples, they both essentially fail even for the simple linear regression case. The use of conditional independence relations seems important for high-dimensional inference.

For MNR, since the essential conditions are (8)-(10), a variable screening-based algorithm will also work under appropriate conditions. Based on this observation, we propose Algorithm S1, which together with some 
numerical results are presented in the Supplementary Material. Compared to Algorithm 1, Algorithm S1 can be substantially faster but the resulting confidence intervals can be a little wider; that is, Algorithm S1 is an accuracy/efficiency trade-off version of Algorithm 1 .

Finally, we note that there are indeed scenarios that conditions (8)-(10) are violated. For example, if all the features are equally correlated or there are a few features whose Markov blanket is of size $O(\sqrt{n})$ or larger, then the condition (8) will be violated, as the algorithm always restricts the Markov blanket to be of size $o(\sqrt{n})$ or smaller. Similarly, if the true model is of size $O(\sqrt{n})$ or larger, then condition (9) will be violated. These conditions can also be violated by the algorithms used for Markov blanket estimation or variable selection, particularly when the sample size is small. The screening property is itself a large sample property. Our numerical experience shows that the MNR method is pretty robust to violations of the conditions (8) -(10). This will be demonstrated in Section 3.4 .

\subsection{Generalized Linear Models}

The MNR method can be easily extended to the generalized linear models (GLMs) whose density function is given in the canonical form

$$
f(y \mid \boldsymbol{x}, \boldsymbol{\beta})=\exp (\vartheta y-b(\vartheta)+c(y))
$$

where $b(\cdot)$ is continuously differentiable, and $\vartheta$ is the natural parameter relating $y$ to the features $\boldsymbol{x}$ via a linear function $\vartheta=\beta_{0}+x_{1} \beta_{1}+\cdots+x_{p} \beta_{p}$. This class of GLMs includes Poisson regression, logistic regression and linear regression (with known variance). Note that for Cox proportional hazards models, the parameters can be estimated by maximizing the partial likelihood function (Cox, 1975), based on which the Cox regression can be converted to a Poisson regression. See, e.g., Chapter 13 of McCullagh and Nelder (1989) for the detail. This conversion is important, which enables the use of the MNR method for high-dimensional survival data analysis.

To justify this extension, we establish the following lemma, where we assume that the features follow a multivariate normal distribution $N(0, \Sigma)$ and each has been standardized to have a mean 0 and variance 1 . The proof follows the same logic as that of Lemma 1, but the precision matrix involved in Lemma 1 is replaced by the inverse of the Fisher information matrix of the GLM. Refer to the Supplementary Material for the detail.

Lemma 2. Let $\hat{\xi}_{j} \supseteq \xi_{j}$ denote any Markov neighborhood of feature $x_{j}$, let $\hat{\boldsymbol{S}}_{*} \supseteq \boldsymbol{S}_{*}$ denote any reduced feature space, and let $D_{j}=\{j\} \cup \hat{\xi}_{j} \cup \hat{\boldsymbol{S}}_{*}$. Consider a subset $G L M$ with the features $\boldsymbol{X}_{D_{j}}$, let $\hat{\boldsymbol{\beta}}_{D_{j}}$ denote the MLE of $\boldsymbol{\beta}_{D_{j}}$, and let $\hat{\beta}_{j}$ denote the component of $\hat{\boldsymbol{\beta}}_{D_{j}}$ corresponding to feature $X_{j}$. If $\left|D_{j}\right|=o\left(n^{1 / 2}\right)$, then, as $n \rightarrow \infty$, the following results hold:

(i) $\sqrt{n}\left(\hat{\beta}_{j}-\beta_{j}\right) \sim N\left(0, k_{j j}\right)$, where $k_{j j}$ denotes the $(j, j)$-th entry of the inverse of the Fisher information matrix $K=I^{-1}=\left[E\left(b^{\prime \prime}\left(\boldsymbol{x}^{T} \boldsymbol{\beta}\right) \boldsymbol{x} \boldsymbol{x}^{T}\right)\right]^{-1}$, and $\boldsymbol{\beta}$ denotes the true regression coefficients.

(ii) $\sqrt{n}\left(\hat{\beta}_{j}-\beta_{j}\right) / \sqrt{\hat{k}_{j j}} \sim N(0,1)$, where $\hat{k}_{j j}$ denotes the $(j, j)$-th entry of the inverse of the observed information matrix $J_{n}\left(\hat{\boldsymbol{\beta}}_{D_{j}}\right)=-\sum_{i=1}^{n} H_{\hat{\boldsymbol{\beta}}_{D_{j}}}\left(\log f\left(y_{i} \mid \boldsymbol{\beta}_{D_{j}}, \boldsymbol{x}_{D_{j}}\right)\right) / n$ and $H_{\hat{\boldsymbol{\beta}}_{D_{j}}}(\cdot)$ denotes the Hessian matrix evaluated at the $M L E \hat{\boldsymbol{\beta}}_{D_{j}}$.

Lemma 2 implies that the estimate, $p$-value and confidence interval of $\beta_{j}$ can be calculated from the subset GLM as in conventional low-dimensional GLMs. For GLMs, variable selection can be done using the SCAD, MCP or Lasso algorithm, and variable screening can be done using the sure independence screening algorithm 
developed in Fan and Song (2010). By Theorem 5 of Fan and Song (2010), we can bound the size of $\hat{\boldsymbol{S}}_{*}$ by $O\left(n^{\frac{1}{2}-\frac{\varepsilon}{2}}\right)$ for a small constant $\varepsilon>0$ with a slight modification of the technical conditions therein. Therefore, the theorems parallel to Theorem 1 and Theorem S1 (in the Supplementary Material) can be proved for GLMs. For simplicity, they are omitted in the paper.

\subsection{Joint Inference}

The MNR method described above deals with only one coefficient $\beta_{j}$ in each subset regression. In fact, it can be easily extended to conduct joint inference for several coefficients. Let $\boldsymbol{A} \subset \boldsymbol{V}$ denote a set of features for which the joint inference for the corresponding coefficients is desired. Define $\xi_{\boldsymbol{A}}=\cup_{j \in \boldsymbol{A}} \xi_{j}$ as the union of the Markov blankets of the features in $\boldsymbol{A}$. Let $\boldsymbol{M}=\boldsymbol{A} \cup \hat{\xi}_{\boldsymbol{A}} \cup \hat{\boldsymbol{S}}_{*}$. Then a subset regression can be conducted with the features included in $\boldsymbol{M}$. For high-dimensional linear regression, if $|\boldsymbol{M}|=O\left(n^{1 / 2}\right)$, then, similar to Theorem 1, we can show $\sqrt{n}\left(\hat{\boldsymbol{\beta}}_{A}-\boldsymbol{\beta}_{A}\right) \sim N\left(0, \sigma^{2} \Theta_{A A}\right)$, where $\Theta_{A A}$ denotes the submatrix of the precision matrix $\Theta$ constructed by its $A$ rows and $A$ columns. Similarly, for high-dimensional GLMs, we can show $\sqrt{n}\left(\hat{\boldsymbol{\beta}}_{A}-\boldsymbol{\beta}_{A}\right) \sim N\left(0, K_{A A}\right)$, where $K_{A A}$ denotes the submatrix of $K=\left[E\left(b^{\prime \prime}\left(\boldsymbol{x}^{T} \boldsymbol{\beta}\right) \boldsymbol{x} \boldsymbol{x}^{T}\right]^{-1}\right.$ constructed by its $A$ rows and $A$ columns.

\section{Simulation Studies}

\subsection{A Conceptual Experiment}

We first test the concept of MNR using a large- $n$-small- $p$ example; that is, whether the confidence intervals generated by MNR coincide with those generated by the OLS method as the sample size $n$ becomes large. We generated a dataset from the model (3) with $n=2000$ and $p=50$, where $\sigma^{2}$ was set to 1 , the covariates $\boldsymbol{X}$ were generated from a zero-mean multivariate Gaussian distribution with a Toeplitz covariance matrix given by $\Sigma_{i, j}=$ $0.9^{|i-j|}$ for $i, j=1, \ldots, p$, and the true regression coefficients $\left(\beta_{0}, \beta_{1}, \beta_{2}, \ldots, \beta_{5}\right)=(1,0.2,0.4,-0.3,-0.5,1.0)$ and $\beta_{6}=\cdots=\beta_{p}=0$.

Figure 1 compares the $95 \%$ confidence intervals of $\beta_{1}, \ldots, \beta_{p}$ produced by MNR and OLS with the simulated dataset. For MNR, the nodewise regression algorithm (with SIS-Lasso performed for each node) was employed for Markov blanket estimation, and SIS-SCAD was employed for variable selection. The SIS-Lasso refers to a variable selection procedure implemented in the package SIS (Saldana and Feng, 2018), where the sure independence screening (SIS) algorithm (Fan and Lv, 2008) was first applied for variable screening and then the Lasso algorithm was applied to select variables from those survived from the screening procedure. The SIS-SCAD and SIS-MCP can be interpreted in the same way. As expected from Theorem 1, OLS and MNR produced almost identical confidence intervals for each regression coefficient. In this simulation, we set $n$ excessively large, which ensures the convergence of the sample covariance matrix to the true covariance matrix $\Theta^{-1}$. In fact, MNR can work well with a smaller value of $n$ as illustrated by the following small $n$-large- $p$ examples.

\subsection{An Illustrative Example}

To illustrate the performance of MNR, we generated 100 independent datasets from the regression (3), where $n=200, p=500, \sigma^{2}=1$, the features were generated from a zero-mean multivariate Gaussian distribution with 


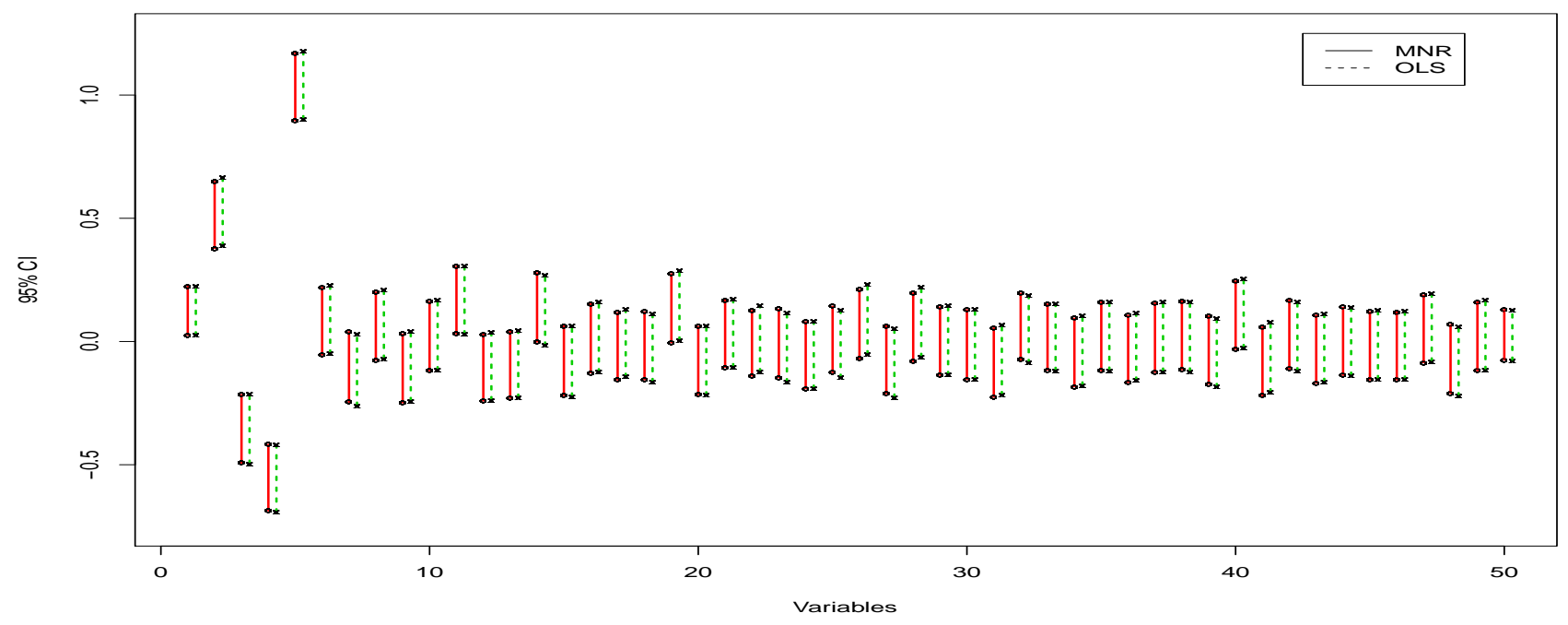

Figure 1: The $95 \%$ confidence intervals of $\beta_{1}, \ldots, \beta_{p}$ produced by the MNR (solid line) and OLS (dashed line) methods for a dataset with $n=2000$ and $p=50$.

a Toeplitz covariance matrix given by $\Sigma_{i, j}=0.9^{|i-j|}$ for $i, j=1, \ldots, p$, and the true regression coefficients were given by $\left(\beta_{0}, \beta_{1}, \beta_{2}, \ldots, \beta_{5}\right)=(1,2,4,-3,-5,10)$ and $\beta_{6}=\cdots=\beta_{p}=0$. We note that the same covariance matrix has been used in van de Geer et al. (2014) to illustrate the performance of desparsified-Lasso. For convenience, we call this model a Toeplitz-covariance linear regression model.

\subsubsection{Illustration of MNR}

Algorithm 1 was run for this example as in Section [3.1, i.e., applying SIS-SCAD for variable selection and the nodewise regression algorithm for Markov blanket estimation. Table 1 summarizes the coverage rates and widths of the $95 \%$ confidence intervals produced by MNR for each regression coefficient. For the non-zero regression coefficients (denoted by "signal"), the mean coverage rate and mean width of the confidence intervals are defined, respectively, by

$$
\bar{p}_{\text {cover }}=\sum_{j=1}^{100} \sum_{i \in \boldsymbol{S}_{*}} \hat{p}_{i}^{(j)} /\left(100 \cdot\left|\boldsymbol{S}_{*}\right|\right), \quad \bar{w}_{\mathrm{CI}}=\sum_{j=1}^{100} \sum_{i \in \boldsymbol{S}_{*}} \hat{w}_{i}^{(j)} /\left(100 \cdot\left|\boldsymbol{S}_{*}\right|\right),
$$

and their respective standard deviations are defined by

$$
\begin{aligned}
\sigma\left(\bar{p}_{\text {cover }}\right) & =\sqrt{\operatorname{Var}\left\{\hat{p}_{i}^{(j)}: i \in \boldsymbol{S}_{*}, j=1,2, \ldots, 100\right\} / 100} \\
\sigma\left(\bar{w}_{\mathrm{CI}}\right) & =\sqrt{\operatorname{Var}\left\{\hat{w}_{i}^{(j)}: i \in \boldsymbol{S}_{*}, j=1,2, \ldots, 100\right\} / 100}
\end{aligned}
$$

where $\hat{w}_{i}^{(j)}$ denotes the width of the $95 \%$ confidence interval of $\beta_{i}$ constructed with the $j$ th dataset, $\hat{p}_{i}^{(j)} \in\{0,1\}$ indicates the coverage of $\beta_{i}$ by the confidence interval, and $\operatorname{Var}\{\cdot\}$ denotes the variance. By dividing by 100 in (14), the standard deviation represents the variability of the mean value (averaged over 100 independent 
datasets) for a single regression coefficient. For the zero regression coefficients (denoted by "noise"), the mean coverage rate, the mean width, and their standard deviations can be defined similarly.

For comparison, we applied the desparsified Lasso, ridge projection and multi-split methods to this example. These methods have been implemented in the $R$ package $h d i$ (Meier et al.,2016). The comparison indicates that MNR significantly outperforms the existing methods: for both the non-zero and zero regression coefficients, the mean coverage rates produced by MNR are much closer to their nominal level. The reason why desparsifiedLasso suffers from coverage deficiency for non-zero regression coefficients will be explained in Section 3.3.1

Table 1: Coverage rates and widths of the 95\% confidence intervals produced by MNR with Algorithm 1 for the Toeplitz-covariance linear regression model, where "signal" and "noise" denote non-zero and zero regression coefficients, respectively. For "signal", the reported mean value and standard deviation (in the parentheses) are defined in (13) and (14), respectively. For "noise", they are defined similarly.

\begin{tabular}{cccccc}
\hline Measure & & Desparsified-Lasso & Ridge & Multi-Split & MNR \\
\hline \multirow{2}{*}{ Coverage } & signal & $0.384(0.049)$ & $0.576(0.049)$ & $0.202(0.040)$ & $0.956(0.021)$ \\
& noise & $0.965(0.018)$ & $0.990(0.010)$ & $1.000(6.4 \mathrm{e}-4)$ & $0.950(0.022)$ \\
\hline \multirow{2}{*}{ Width } & signal & $0.673(0.005)$ & $1.086(0.010)$ & $2.711(0.097)$ & $0.822(0.011)$ \\
& noise & $0.691(0.005)$ & $1.143(0.008)$ & $2.790(0.103)$ & $0.869(0.007)$ \\
\hline
\end{tabular}

As discussed previously, MNR converts the problem of variable selection as a multiple hypothesis testing problem. To illustrate the potential of MNR in variable selection, we converted the $p$-values produced by the subset regressions to z-scores using the inverse probability integral transformation

$$
Z_{i}^{(j)}=\Phi^{-1}\left(1-q_{i}^{(j)}\right), \quad i=1,2, \ldots, p, \quad j=1,2, \ldots, 100,
$$

where $q_{i}^{(j)}$ denotes the $p$-value calculated via subset regression for feature $i$ with dataset $j$, and $\Phi(\cdot)$ denotes the CDF of the standard Gaussian distribution. Figure S1 (in the Supplementary Material) shows the histogram of the z-scores, which indicates that the true and false features can be well separated by the z-scores. The empirical Bayesian method developed by Liang and Zhang (2008) was applied to each of the 100 datasets for simultaneously testing the hypotheses (11). At a FDR level of $q=0.0001$, which is measured by the q-value of Storev (2002), the method led to exact identifications of the true and false features for all 100 datasets, i.e., both the false selection rate (FSR) and negative selection rate (FSR) are 0. More results were shown in Table 2. Here the FSR and NSR are defined by

$$
F S R=\frac{\sum_{j=1}^{100}\left|\hat{\boldsymbol{S}}_{j} \backslash \boldsymbol{S}_{*}\right|}{\sum_{j=1}^{100}\left|\hat{\boldsymbol{S}}_{j}\right|}, \quad N S R=\frac{\sum_{j=1}^{100}\left|\boldsymbol{S}_{*} \backslash \hat{\boldsymbol{S}}_{j}\right|}{\sum_{j=1}^{100}\left|\boldsymbol{S}_{*}\right|},
$$

where $\boldsymbol{S}_{*}$ is the set of true features, and $\hat{\boldsymbol{S}}_{j}$ is the set of selected features for dataset $j$. For comparison, SIS-SCAD, SIS-MCP and SIS-Lasso were applied to these datasets for performing variable selection under their default settings in the package SIS. Table 2 shows that MNR can significantly outperform the existing methods in high-dimensional variable selection. As mentioned previously, compared to the existing methods, a significant advantage of the MNR-based variable selection method is that it controls the FDR of selected features. 
Table 2: Variable selection for the Toeplitz-covariance linear regression with the MNR, SIS-SCAD, SIS-MCP and SIS-Lasso methods.

\begin{tabular}{cccccccc}
\hline & \multicolumn{5}{c}{ MNR } & & \\
\cline { 2 - 5 } Measure & $q=0.0001$ & $q=0.001$ & $q=0.01$ & SIS-SCAD & SIS-MCP & SIS-Lasso \\
\hline FSR & 0 & 0.004 & 0.022 & 0.127 & 0.175 & 0.819 \\
NSR & 0 & 0 & 0 & 0 & 0 & 0 \\
\hline
\end{tabular}

\subsubsection{Illustration of Joint Inference with MNR}

To illustrate the use of MNR for joint inference, we constructed Bonferroni joint confidence intervals based on the subset regression for each of the following sets of parameters: $\left(\beta_{1}, \beta_{2}\right),\left(\beta_{3}, \beta_{4}, \beta_{5}\right),\left(\beta_{1}, \beta_{6}\right),\left(\beta_{7}, \beta_{10}\right)$, and $\left(\beta_{20}, \beta_{200}, \beta_{400}\right)$, which have covered the cases of combinations of nonzero coefficients, combinations of zero and nonzero coefficients, and combinations of zero coefficients. For each set of parameters, as described in Section 2.3, the subset regression was constructed by unioning the Markov neighborhoods of the corresponding features, and then the $95 \%$ joint confidence intervals for the set of parameters were constructed using the standard Bonferroni method. The Markov neighborhood of each feature was constructed as in Section 3.1 using nodewise regression for GGM estimation and SIS-SCAD for variable selection. Table 3 summarizes the coverage rates of the joint confidence intervals, and it indicates that the proposed method works reasonably well for this example.

Table 3: Coverage rates of $95 \%$ joint confidence intervals produced by MNR for the set of parameters: $\left(\beta_{1}, \beta_{2}\right)$, $\left(\beta_{3}, \beta_{4}, \beta_{5}\right),\left(\beta_{1}, \beta_{6}\right),\left(\beta_{7}, \beta_{10}\right)$, and $\left(\beta_{20}, \beta_{200}, \beta_{400}\right)$, where the number in the parentheses represents the standard deviation of the joint coverage rate averaged over 100 independent datasets.

\begin{tabular}{cccccc}
\hline Parameters & $\left(\beta_{1}, \beta_{2}\right)$ & $\left(\beta_{3}, \beta_{4}, \beta_{5}\right)$ & $\left(\beta_{1}, \beta_{6}\right)$ & $\left(\beta_{7}, \beta_{10}\right)$ & $\left(\beta_{20}, \beta_{200}, \beta_{400}\right)$ \\
\hline Joint coverage rate & $0.97(0.017)$ & $0.95(0.022)$ & $0.93(0.026)$ & $0.97(0.017)$ & $0.93(0.026)$ \\
\hline
\end{tabular}

\subsection{Simulation Studies with More Regression Models}

\subsubsection{Linear Regression}

We simulated 100 independent datasets from the linear regression (3) where $n=200, p=500, \sigma^{2}=1$, the features were generated from a zero-mean Gaussian distribution with the precision matrix $\Sigma^{-1}=\Theta=\left(\theta_{i j}\right)$ given by

$$
\theta_{i j}= \begin{cases}0.5, & \text { if }|j-i|=1, i=2, \ldots,(p-1), \\ 0.25, & \text { if }|j-i|=2, i=3, \ldots,(p-2), \\ 1, & \text { if } i=j, i=1, \ldots, p \\ 0, & \text { otherwise }\end{cases}
$$

and the regression coefficients were given by $\left(\beta_{0}, \beta_{1}, \beta_{2}, \ldots, \beta_{5}\right)=(1,2,2.5,3,3.5,4)$ and $\beta_{6}=\cdots=\beta_{p}=0$. Since the precision matrix has an autoregressive (AR) structure, for convenience, we call this model an $\operatorname{AR}(2)$ - 
Table 4: Coverage rates and widths of the $95 \%$ confidence intervals produced by MNR, desparsified-Lasso, and ridge projection for the $\mathrm{AR}(2)$-precision linear, logistic and Cox regression. Refer to the caption of Table 1 for the notation.

\begin{tabular}{|c|c|c|c|c|c|}
\hline Response & Measure & & Desparsified-Lasso & Ridge & MNR \\
\hline \multirow{4}{*}{ Gaussian } & \multirow{2}{*}{ Coverage } & signal & $0.2300(0.0421)$ & $0.3340(0.0447)$ & $0.9500(0.0218)$ \\
\hline & & noise & $0.9640(0.0186)$ & $0.9922(0.0088)$ & $0.9503(0.0217)$ \\
\hline & \multirow{2}{*}{ Width } & signal & $0.2810(0.0027)$ & $0.4481(0.0043)$ & $0.2806(0.0022)$ \\
\hline & & noise & $0.2723(0.0024)$ & $0.4335(0.0036)$ & $0.2814(0.0024)$ \\
\hline \multirow{4}{*}{ Binary } & \multirow{2}{*}{ Coverage } & signal & $0.004(0.0063)$ & $0(0)$ & $0.9320(0.0252)$ \\
\hline & & noise & $0.9953(0.0068)$ & $1.0(4.5 \mathrm{e}-4)$ & $0.9373(0.0242)$ \\
\hline & \multirow{2}{*}{ Width } & signal & $0.6424(0.0101)$ & $1.0775(0.0110)$ & $1.9473(0.0529)$ \\
\hline & & noise & $0.5782(0.0081)$ & $1.0100(0.0095)$ & $0.9799(0.0132)$ \\
\hline \multirow{4}{*}{ Survival } & \multirow[b]{2}{*}{ Coverage } & signal & - & - & $0.9140(0.0281)$ \\
\hline & & noise & - & - & $0.9354(0.0246)$ \\
\hline & \multirow{2}{*}{ Width } & signal & - & - & $0.3356(0.0018)$ \\
\hline & & noise & - & - & $0.2683(0.0017)$ \\
\hline
\end{tabular}

precision linear regression model.

Algorithm 1 was first applied to this example with the numerical results summarized in Table 4 where the $\psi$-learning algorithm was employed for Markov blanket estimation, and SIS-MCP was employed for variable selection. The $\psi$-learning algorithm has been implemented in the R-package equSA (Jia et al., 2018). It provides an equivalent measure of the partial correlation coefficient, the so-called $\psi$-partial correlation coefficient, for estimating Gaussian graphical models under the small- $n$-large- $p$ scenario. The algorithm consists of two screening stages. The first stage is correlation screening, which, via a multiple hypothesis test for correlation coefficients, determines for each feature a conditioning set used for calculating the $\psi$-partial correlation coefficient. The second stage is $\psi$-partial correlation screening, which, via a multiple hypothesis test for $\psi$-partial correlation coefficients, determines the Gaussian graphical model. Corresponding to the two stages, the algorithm consists of two tuning parameters, $\alpha_{1}$ and $\alpha_{2}$, which specify the significance levels of the two multiple hypothesis tests, respectively. In all applications of the $\psi$-learning algorithm in this paper, we set $\alpha_{1}=0.1$ and $\alpha_{2}=0.05$ as suggested by Liang et al. (2015). In general, $\alpha_{1}$ should be slightly large to avoid potential loss of important features in the conditioning set of each feature. The nodewise regression algorithm has also been applied to this example for Markov blanket estimation, and the results are similar.

For comparison, the desparsified Lasso and ridge projection methods were applied to this example. Both methods have been implemented in the $R$ package $h d i$ (Meier et al., 2016). The multi-split method is also available in $h d i$, but it often suffered from a convergence issue in applications to this example and thus not included for comparison. Table 4 shows that MNR significantly outperforms the existing methods: The coverage 
rates produced by MNR are almost identical to their nominal levels for both zero and non-zero regression coefficients; while the coverage rates produced by the other methods are far from their nominal levels, especially for the nonzero regression coefficients.

For the non-zero regression coefficients, the confidence intervals produced by desparsified-Lasso have about the same widths as those by MNR, but the former have much lower coverage rates. The coverage deficiency of desparsified-Lasso are due to at least two reasons: (i) the bias-corrected estimator $\hat{\boldsymbol{\beta}}_{b c}$ is still biased; and (ii) the required sparsity condition is violated. The bias of $\hat{\boldsymbol{\beta}}_{b c}$ can be easily seen from the derivation procedure of $\hat{\boldsymbol{\beta}}_{b c}$, which is due to Zhang and Zhang (2014). Let $Z_{j}$ denote the residual of the regression $X_{j}$ versus all other features $\boldsymbol{X}[-j]$, and let $P_{j k}=X_{k}^{T} Z_{j} / X_{j}^{T} Z_{j}$. Then the following identity holds

$$
\frac{Y^{\prime} Z_{j}}{X_{j}^{T} Z_{j}}=\beta_{j}+\sum_{k \neq j} P_{j k} \beta_{k}+\frac{\epsilon^{\prime} Z_{j}}{X_{j}^{T} Z_{j}}
$$

where $Y$ and $\epsilon$ are as defined in (3) . Plugging the Lasso estimator $\hat{\boldsymbol{\beta}}_{\text {Lasso }}$ (of the regression $Y$ versus $\boldsymbol{X}$ ) into (17) leads to the bias-corrected estimator

$$
\hat{\beta}_{b c, j}=\frac{Y^{\prime} Z_{j}}{X_{j}^{T} Z_{j}}-\sum_{k \neq j} P_{j k} \hat{\beta}_{\text {Lasso }, k}=\hat{\beta}_{\text {Lasso }, j}+Z_{j}^{\prime}\left(Y-\boldsymbol{X} \hat{\boldsymbol{\beta}}_{\text {Lasso }}\right) / Z_{j}^{\prime} X_{j}, \quad j=1,2, \ldots, p,
$$

which is essentially the same with the estimator given in (11). Here $\hat{\beta}_{b c, j}$ and $\hat{\beta}_{\text {Lasso,j }}$ denote the $j$-th component of $\hat{\boldsymbol{\beta}}_{b c}$ and $\hat{\boldsymbol{\beta}}_{\text {Lasso }}$, respectively. The $\hat{\boldsymbol{\beta}}_{b c}$ can have the bias of $\hat{\boldsymbol{\beta}}_{\text {Lasso }}$ much corrected. However, as implied by (17), $\hat{\boldsymbol{\beta}}_{b c}$ is still generally biased because the Lasso estimator $\hat{\boldsymbol{\beta}}_{\text {Lasso }}$ is generally biased. Such a biased estimator shifts the center of the confidence interval and thus leads to the coverage deficiency problem. For the error term $\Delta_{n}$ defined in (2), Dezeure et al. (2015) proved that it is negligible if the sparsity condition $\left|\boldsymbol{S}_{*}\right|=o(\sqrt{n} / \log (p))$ holds, the precision matrix is row-sparse at a level of $o(n / \log (p))$, and some other regularity conditions on the design matrix hold. Among these conditions, the model sparsity condition $\left|\boldsymbol{S}_{*}\right|=o(\sqrt{n} / \log (p))$ is a little restrictive and can be easily violated. For example, for a problem with $\left|\boldsymbol{S}_{*}\right|=5$ and $p=500$, the sample size $n$ should be at least a few thousands to satisfy the condition $s_{0} \ll \sqrt{n} / \log (p)$. As the result, the error term $\Delta_{n}$ might not be negligible, which can also cause the coverage deficiency issue. Since $\left\|\hat{\boldsymbol{\beta}}_{\text {Lasso }}-\boldsymbol{\beta}\right\|_{1}=$ $O_{p}\left(\left|\boldsymbol{S}_{*}\right| \sqrt{\log (p) / n}\right.$ ) (Dezeure et al., 2015), violation of the sparsity condition also worsens the bias of $\hat{\boldsymbol{\beta}}_{b c}$. We note that the model sparsity condition $\left|\boldsymbol{S}_{*}\right|=o(\sqrt{n})$ required by MNR is much weaker than $\left|\boldsymbol{S}_{*}\right|=o(\sqrt{n} / \log (p))$ under the small- $n$-large- $p$ scenario.

In our numerical experience, the coverage deficiency of desparsified-Lasso is mainly due to the bias of $\hat{\boldsymbol{\beta}}_{b c}$. We illustrate this issue using two simulation studies. The first one is given as follows and the other one is given in Section 3.4. In Table 5, we reported the values of $\hat{\boldsymbol{\beta}}_{b c, j}$ 's, $j=1,2, \ldots, 8$, for the $\mathrm{AR}(2)$-precision linear regression. It is easy to see that the desparsified-Lasso estimate is severely biased for the nonzero coefficients $\beta_{1}, \ldots, \beta_{5}$, which significantly shifts the centers of the resulting confidence intervals and thus leads to the coverage deficiency problem. Note that $\hat{\beta}_{b c, 6}$ is also biased due to the strong correlation between $X_{6}$ and $X_{5}$. For comparison, we included in Table 5 the MNR estimates of these coefficients, which are unbiased for both zero and nonzero coefficients. 
Table 5: Regression coefficient estimates (averaged over 100 independent datasets) produced by MNR and desparsified-Lasso for the $\mathrm{AR}(2)$-precision linear regression (with $\left|\boldsymbol{S}_{*}\right|=5, p=500$ and $n=200$ ), where the numbers in the parentheses represent the standard deviations of the estimates.

\begin{tabular}{cccccccccc}
\hline Method & Measure & $\beta_{1}$ & $\beta_{2}$ & $\beta_{3}$ & $\beta_{4}$ & $\beta_{5}$ & $\beta_{6}$ & $\beta_{7}$ & $\beta_{8}$ \\
\hline \multirow{2}{*}{ desparsified } & true & 2 & 2.5 & 3 & 3.5 & 4 & 0 & 0 & 0 \\
\hline \multirow{2}{*}{ MNR } & $\hat{\boldsymbol{\beta}}_{b c}$ & 1.841 & 2.274 & 2.698 & 3.270 & 3.849 & -0.051 & -0.007 & 0.016 \\
& $\mathrm{SD}$ & $(0.008)$ & $(0.009)$ & $(0.009)$ & $(0.007)$ & $(0.007)$ & $(0.006)$ & $(0.007)$ & $(0.007)$ \\
\hline & $\hat{\boldsymbol{\beta}}_{M N R}$ & 1.997 & 2.503 & 2.994 & 3.498 & 4.001 & 0.014 & 0.004 & -0.002 \\
& $\mathrm{SD}$ & $(0.006)$ & $(0.008)$ & $(0.008)$ & $(0.007)$ & $(0.006)$ & $(0.007)$ & $(0.008)$ & $(0.008)$ \\
\hline
\end{tabular}

\subsubsection{Logistic Regression}

We simulated 100 datasets for a logistic regression. For each dataset, we set $n=300, p=500,\left(\beta_{0}, \beta_{1}, \ldots, \beta_{5}\right)=$ $(1,2,2.5,3,3.5,4), \beta_{6}=\cdots=\beta_{p}=0$, and generated the covariates from a zero-mean multivariate Gaussian distribution with the precision matrix given by (16). For convenience, we call this model an $\operatorname{AR}(2)$ precision logistic regression model. Each dataset consisted of 150 case samples and 150 control samples. To alleviate the convergence issues suffered by the GLM estimation procedure $g l m$ in R, we set $n$ slightly large for this example.

Algorithm 1 was run for the datasets, where the SIS-MCP algorithm was employed for variable selection and the $\psi$-learning algorithm was employed for Markov blanket estimation. The nodewise regression algorithm was also applied for Markov blanket estimation, the results were similar. The numerical results were summarized in Table 4, which indicates that MNR significantly outperforms the other methods. Desparsified-Lasso and ridge projection essentially fail for this example.

\subsubsection{Cox Regression}

For Cox regression, which is also known as Cox proportional-hazards model, we let $\lambda(t)$ denote the hazard rate at time $t$ and let $\lambda_{0}(t)$ denote the baseline hazard rate. The Cox model can then be expressed as

$$
\lambda(t)=\lambda_{0}(t) \exp \left(\beta_{1} X_{1}+\beta_{2} X_{2}+\ldots+\beta_{p} X_{p}\right)
$$

In the simulation, we set $\left(\beta_{1}, \ldots, \beta_{5}\right)=(1,1,1,1,1), \beta_{6}=\cdots=\beta_{p}=0$, the baseline hazard rate $\lambda_{0}(t)=$ $\lambda_{0}=0.1$, and the censoring hazard rate $\lambda_{c}=1$; generated the event time from the Weibull distribution with the shape parameter $=1$ and the scale parameter $=\lambda_{0} \exp \left(-\sum_{i=1}^{p} X_{i} \beta_{i}\right)$; generated the censoring time from the Weibull distribution with the shape parameter $=1$ and the scale parameter $=\lambda_{c}$; set the observed survival time as the minimum of the event time and the censoring time for each subject; and generated the features $X_{1}, \ldots, X_{p}$ from a zero-mean multivariate normal distribution with the precision matrix given by (16). For convenience, we call this model an AR(2)-precision Cox regression model. We simulated 100 datasets from this model with $n=300$ and $p=500$.

Algorithm 1 was run for the datasets, where the SIS-Lasso algorithm was used for variable selection and the $\psi$-learning algorithm was used for Markov blanket estimation. The numerical results were summarized in 
Table 4. The nodewise regression algorithm was also applied for Markov blanket estimation, the results were similar. In the MNR results, we can observe some slight bias, which mainly comes from the model selection error and the estimation error of the Markov blankets. Our numerical experience shows that the nominal level can be reached by MNR with the correct model and correct Markov neighborhoods or when $n$ becomes large.

In addition to coverage rates, Table 4 reports mean widths of the confidence intervals resulted from different methods. For linear regression, the confidence intervals by MNR are narrower than those by ridge projection, and of about the same width as those by desparsified-Lasso. However, as analyzed previously, desparsifiedLasso often suffers from the coverage deficiency issue. For logistic and Cox regression, the comparison is not meaningful, as the other methods either fail or are not available.

To explore the potential of MNR in variable selection, we have calculated z-scores in (15) based on the $p$ values generated by MNR for the datasets simulated above. Figures S2-S4 show the histograms of the z-scores, which indicate that the true and false features can always be well separated by z-scores for all these datasets. This is an attractive feature of MNR and its use for feature selection will be further explored in Section 4 .

\subsection{Robustness of Markov Neighborhood Regression}

This section studies the robustness of MNR to violations of the conditions (8)-(10). This issue has been partially studied in Section 2.2 of the Supplementary Material, where the condition (9) is violated when the size of $\hat{\boldsymbol{S}}_{*}$ is restricted to 3 . Recall that for the Toeplitz-covariance regression, we have $\left|\boldsymbol{S}_{*}\right|=5,\left|\xi_{j}\right|=2$ for $j=2,3, \ldots, p-1$, and $\left|\xi_{j}\right|=1$ for $j=1$ and $p$. Therefore, setting $\left|\hat{\boldsymbol{S}}_{*}\right|=3$ leads to some true features missed in each subset regression. As shown in Table S1 (in the Supplementary Material), this results in wider confidence intervals for both zero and nonzero regression coefficients, although the coverage rates are not much affected.

In what follows, we consider one linear regression example where all features are equally correlated with a correlation coefficient of 0.8 . The features were generated from a zero-mean multivariate Gaussian distribution with the covariance matrix given by

$$
\Sigma_{i, j}=0.8, \quad \text { for all } i \neq j, \quad \Sigma_{i, i}=1 \quad \text { for all } i .
$$

We set $p=500, n=300,\left(\beta_{0}, \beta_{1}, \ldots, \beta_{10}\right)=(1,2,2.5,3,3.5,4,5,6,7,-8,-9)$, and $\beta_{11}=\cdots=\beta_{p}=0$, and generated 100 independent datasets in total. For convenience, we will call this model an equi-correlation linear regression model. The same model has been used in van de Geer et al. (2014) to illustrate the performance of desparsified-Lasso, but with different sample sizes and regression coefficients. For this example, it is easy to see that for each feature $x_{j}$, the Markov blanket $\xi_{j}$ consists of all other $p-1$ features. That is, the condition (8) is violated, as we always restrict the Markov blanket to be much smaller than $p$.

Algorithm 1 was first applied to this example, where SIS-MCP was used for variable selection, and nodewise regression (with SIS-Lasso performed for each node) was used for Markov blanket estimation. All the algorithms were run under their default setting in the R package SIS. For comparison, desparsified-Lasso and ridge projection methods were also applied to this example. Both methods were run under their default settings in the $\mathrm{R}$ package $h d i$. The numerical results were summarized in Table 6, which indicates that MNR is pretty robust to the misspecification of the Markov blanket for this example. In terms of mean coverage rates and widths, MNR produced most accurate confidence intervals compared to the desparsified-Lasso and ridge projection methods. 
Table 6: Coverage rates and widths of the $95 \%$ confidence intervals produced by desparsified-Lasso, ridge projection and MNR (with Algorithm 1) for the equi-correlation linear regression model. Refer to the caption of Table 1 for the notation.

\begin{tabular}{ccccc}
\hline Measure & - & Desparsified-Lasso & Ridge & MNR \\
\hline \multirow{2}{*}{ Coverage } & signal & $0.916(0.028)$ & $0.973(0.016)$ & $0.938(0.024)$ \\
& noise & $0.963(0.019)$ & $0.990(0.010)$ & $0.951(0.022)$ \\
\hline \multirow{2}{*}{ Width } & signal & $0.656(0.003)$ & $1.066(0.007)$ & $0.551(0.003)$ \\
& noise & $0.657(0.004)$ & $1.069(0.007)$ & $0.554(0.003)$ \\
\hline
\end{tabular}

Compared to the results reported in Table 4 for the AR(2)-precision linear regression, desparsified-Lasso works much better for this example. For the AR(2)-precision linear regression, Table 5 shows that $\hat{\boldsymbol{\beta}}_{b c}$ is severely biased and thus the method suffers from coverage deficiency for nonzero coefficients. To have this issue further explored, we reported in Table $7 \hat{\boldsymbol{\beta}}_{b c}$ and $\hat{\boldsymbol{\beta}}_{\mathrm{MNR}}$ for the nonzero coefficients $\beta_{1}, \beta_{2}, \ldots, \beta_{10}$. The comparison with the true value shows that $\hat{\boldsymbol{\beta}}_{b c}$ is nearly unbiased for $\beta_{1}, \ldots, \beta_{8}$, although it is systematically smaller than the true value in magnitudes. As the result, desparsified-Lasso produced a good coverage rate for the non-zero coefficients of this example. MNR continuously works well; $\boldsymbol{\beta}_{\mathrm{MNR}}$ is unbiased and accurate for this example.

Table 7: Regression coefficient estimates (averaged over 100 independent datasets) produced by MNR and desparsified-Lasso for the equi-correlation linear regression model (with $\left|\boldsymbol{S}_{*}\right|=10, p=500$ and $n=300$ ), where the numbers in the parentheses represent the standard deviations of the estimates.

\begin{tabular}{cccccccccccc}
\hline Method & & $\beta_{1}$ & $\beta_{2}$ & $\beta_{3}$ & $\beta_{4}$ & $\beta_{5}$ & $\beta_{6}$ & $\beta_{7}$ & $\beta_{8}$ & $\beta_{9}$ & $\beta_{10}$ \\
\hline \multirow{2}{*}{ - } & true & 2 & 2.5 & 3 & 3.5 & 4 & 5 & 6 & 7 & -8 & -9 \\
\hline \multirow{2}{*}{ desparsified } & $\hat{\boldsymbol{\beta}}_{b c}$ & 1.96 & 2.43 & 2.98 & 3.47 & 3.96 & 4.97 & 5.95 & 6.95 & -7.87 & -8.84 \\
& $\mathrm{SD}$ & $(0.02)$ & $(0.02)$ & $(0.02)$ & $(0.02)$ & $(0.02)$ & $(0.02)$ & $(0.02)$ & $(0.02)$ & $(0.02)$ & $(0.02)$ \\
\hline \multirow{2}{*}{ MNR } & $\hat{\boldsymbol{\beta}}_{\mathrm{MNR}}$ & 2.01 & 2.49 & 3.00 & 3.50 & 3.99 & 5.02 & 5.98 & 6.99 & -8.01 & -9.01 \\
& $\mathrm{SD}$ & $(0.01)$ & $(0.01)$ & $(0.02)$ & $(0.01)$ & $(0.02)$ & $(0.02)$ & $(0.02)$ & $(0.01)$ & $(0.02)$ & $(0.02)$ \\
\hline
\end{tabular}

In summary, MNR is robust to misspecification of the Markov neighborhood. It will perform reasonably well as long as for each subset regression, the Markov neighborhood has covered the major contributors of the subset regression, which include the most significant features to the original regression as well as the most correlated features to the target feature of the subset regression.

\subsection{Computational Complexity}

The MNR method consists of three steps, variable selection, Markov blanket estimation, and subset regression. Its computational complexity is typically dominated by the algorithm used for Markov blanket estimation.

For Algorithm 1, if the Lasso algorithm is employed for variable selection, then, by Meinshausen (2007), 
the computational complexity of this step is upper bounded by $O\left(n^{3} p\right)$ under the small- $n$-large- $p$ scenario. Instead of Lasso, this paper employed the SCAD and MCP algorithms for variable selection which have competitive computational complexity with Lasso (Zhang, 2010). If the $\psi$-learning algorithm is used for Markov blanket estimation, then, by Liang et al. (2015), the computational complexity of this step is upper bounded by $O\left(n^{3} p^{2}\right)$. By condition (10), the computational complexity of each subset regression is $O\left(n^{2}\right)$ and thus the total computational complexity of the subset regression step is $O\left(n^{2} p\right)$. Therefore, the total computational complexity of MNR is upper bounded by $O\left(n^{3} p^{2}\right)$. Alternatively, if the nodewise regression algorithm is used for Markov blanket estimation and Lasso is used for the regression on each node/feature, then the computational complexity of this step is also upper bounded by $O\left(n^{3} p^{2}\right)$ as there are $p$ features in total. In this case, the total computational complexity of MNR is also upper bounded by $O\left(n^{3} p^{2}\right)$. If the graphical Lasso is used for Markov blanket estimation, then the total computational complexity of MNR will be $O\left(p^{3}\right)$, as the graphical Lasso has a computational complexity of $O\left(p^{3}\right)$. In a fast implementation of the graphical Lasso algorithm by making use of the block diagonal structure in its solution (Witten et al., 2011), the total computational complexity of MNR can be reduced to $O\left(p^{2+v}\right)$ for some $0<v \leq 1$.

Since desparsified-Lasso employs the nodewise regression algorithm in estimating the precision matrix and correcting the bias of $\hat{\boldsymbol{\beta}}_{\text {Lasso }}$, its computational complexity is upper bounded by $O\left(n^{3} p^{2}\right)$, the same bound as Algorithm [1]

For a dataset generated from the $\mathrm{AR}(2)$-precision linear regression with $p=500$ and $n=200$, Table 8 summarized the CPU time cost by different methods when running with a single thread on an Intel(R) Xeon(R) CPU E5-2660 v3@2.60GHz machine. For MNR, we employed SIS-MCP for variable selection, but different methods for Markov blanket estimation. We note that ridge projection and multi-split can be substantially faster than MNR, although they are often inferior to MNR in numerical performance. For this example, ridge projection and multi-split took about 3.2 and 3.1 CPU seconds, respectively.

Table 8: CPU times (in seconds) cost by different methods for a dataset generated from the AR(2)-precision linear regression with $p=500$ and $n=200$, where $\mathrm{MNR}_{a}, \mathrm{MNR}_{b}, \mathrm{MNR}_{c}$, and $\mathrm{MNR}_{d}$ mean that $\psi$-learning, nodewise regression (with SIS-Lasso), nodewise regression (with SIS-MCP), and nodewise regression (with SIS-SCAD) were used for Markov blanket estimation, respectively.

\begin{tabular}{cccccc}
\hline Methods & Desparsified-Lasso & $\mathrm{MNR}_{a}$ & $\mathrm{MNR}_{b}$ & $\mathrm{MNR}_{c}$ & $\mathrm{MNR}_{d}$ \\
\hline $\mathrm{CPU}(\mathrm{s})$ & 258 & 152 & 230 & 205 & 250 \\
\hline
\end{tabular}

Finally, we note that MNR can be substantially accelerated via parallel computing, for which both the Markov blanket estimation and subset regression steps can be done in an embarrassingly parallel way. As described in Section 3.3.1, the $\psi$-learning algorithm consists of two screening stages, for which both the correlation coefficients and $\psi$-partial correlation coefficients can be calculated in parallel. Refer to Liang et al. (2015) for more discussions on this issue. If the nodewise regression algorithm is used for Markov blanket estimation, its parallel implementation is obvious. 


\section{Causal Structure Discovery for High-Dimensional Regression}

The causal relationship for a pair or more variables refers to a persistent association which is expected to exist in all situations without being affected by the values of other variables. Due to its attractive feature, which does not only allow better explanations for past events but also enables better predictions for the future, causal discovery has been an essential task in many disciplines. Since, for high-dimensional problems, it is difficult and expensive to identify causal relationships through intervention experiments, passively observed data has thus become an important source to be searched for causal relationships. The challenge of causal discovery from observational data lies in the fact that statistical associations detected from observational data are not necessarily causal.

In statistics, the causal relationship or persistent association can be determined using conditional independence tests. For a large set of variables, a pair of variables are considered to have no direct causal relationship if a subset of the remaining variables can be found such that conditioning on this subset of variables, the two variables are independent. Based on conditional independence tests, Spirtes et al. (2000) proposed the famous PC algorithm for learning the structure of causal Bayesian networks. Later, Bühlmann et al. (2010) extended the PC algorithm to high-dimensional variable selection. The extension is called the PC-simple algorithm which can be used to search for the causal structure around the response variable. Note that the causal structure includes all the possible direct causes and effects of the response variable, i.e., all the parents and children in the terminology of directed acyclic graphs (DAGs). For certain problems, we may be able to determine in logic which are for parents and which are for children, although PC-simple cannot tell. An alternative algorithm that can be used for local causal discovery is the HITON-PC algorithm (Aliferis et al., 2010), which is also an extension of the PC algorithm. The major issue with the PC-simple and HITON-PC algorithms is with their time complexity. For both algorithms, in the worst scenario, i.e., when for each of the $p$ features all conditional independence tests of order from 1 to $p-1$ are conducted, the total number of conditional tests is $O\left(p 2^{p}\right)$. Even under the sparsity constraint, the total number of conditional tests can still be of a high order polynomial of p. See Bühlmann et al. (2010) for more discussions on this issue.

In what follows we describe how the causal structure around the response variable can be discovered for high-dimensional regression based on the output of MNR. The proposed algorithm has a much favorable computational complexity, which is $O\left(n^{3 / 2} p\right)$ in all scenarios. For Gaussian, binary and proportional-hazards response data, the MNR method can be described one by one as follows.

\subsection{Gaussian Response}

Assume that $\boldsymbol{Z}=(Y, \boldsymbol{X})$ jointly follows a multivariate Gaussian distribution $N_{p+1}(0, \Sigma)$. To distinguish the notation from that used in previous sections, we let $\boldsymbol{G}_{z}=\left(\boldsymbol{V}_{z}, \boldsymbol{E}_{z}\right)$ denote the graph underlying the joint Gaussian distribution. Let $\zeta_{j}=\xi_{j} \cup \boldsymbol{S}_{*}$, where $\xi_{j}$ denotes the Markov blanket of $X_{j}$ in the graph $\boldsymbol{G}_{z}$. It is easy to see that $\zeta_{j}$ forms a separator of $Y$ and $X_{j}$ in the graph $\boldsymbol{G}_{z}$. Then, under the faithfulness condition for the joint distribution $N_{p+1}(0, \Sigma)$, we can show as in Liang et al. (2015) that $\rho\left(Y, X_{j} \mid \boldsymbol{X}_{\boldsymbol{V} \backslash\{j\}}\right) \neq 0 \Longleftrightarrow \rho\left(Y, X_{j} \mid \boldsymbol{X}_{\zeta_{j}}\right) \neq 0$, where $\rho(\cdot, \cdot \cdot)$ denotes the partial correlation coefficient. The validity of the faithfulness condition is supported by the Lebesgue measure zero argument (Meek, 1995); that is, the problems that violate the faithfulness condition usually correspond to some particular parameter values that form a zero measure set in the space of all possible parameterizations. Further, by the relationship between partial correlations and regression coefficients, see e.g., 
p.436 of Bühlmann and van de Geer (2011), we have $\rho\left(Y, X_{j} \mid \boldsymbol{X}_{\zeta_{j}}\right) \neq 0 \Longleftrightarrow \beta_{j} \neq 0$, where $\beta_{j}$ is the coefficient of $X_{j}$ in the Markov neighborhood regression $Y \sim X_{j}+\boldsymbol{X}_{\zeta_{j}}$. Therefore, the test for $H_{0}: \beta_{j}=0$ versus $H_{1}: \beta_{j} \neq 0$ can be conducted via the Markov neighborhood regression. Given the $p$-values of individual tests, the causal structure around the response variable $Y$ can be determined via a multiple hypothesis test.

Since the problem of causal structure discovery is to identify a small set of variables that have causal or effect relations with the response variable, a simplified version of Algorithm S1 can be used, which avoids to assess the effect of all variables on the response. In the simplified algorithm, the Markov blankets only need to be found for the variables survived from the variable screening step. The simplified algorithm can be described as follows.

Algorithm 2. (Simplified MNR for Causal Structure Discovery)

(a) (Variable screening) Apply a sure independence screening procedure with $Y$ as the response variable and $\boldsymbol{X}$ as features, to obtain a reduced feature set, $\hat{\boldsymbol{S}}_{*} \subseteq\{1, \ldots, p\}$, with the size $\left|\hat{\boldsymbol{S}}_{*}\right|=O(\sqrt{n} / \log (n))$.

(b) (Markov blanket estimation) For each variable $X_{j} \in \hat{\boldsymbol{S}}_{*}$, apply a sure independence screening procedure to obtain a reduced neighborhood $\hat{\xi}_{j} \subseteq\{1, \ldots, p\}$ with the size $\left|\hat{\xi}_{j}\right|=O(\sqrt{n} / \log (n))$.

(c) (Subset Regression) For each feature $X_{j} \in \hat{\boldsymbol{S}}_{*}$, run a subset regression with the features given by $\left\{X_{j}\right\} \cup$ $\boldsymbol{X}_{\hat{\xi}_{j}} \cup \boldsymbol{X}_{\hat{\boldsymbol{S}}_{*}}$. Conduct inference for $\beta_{j}$, including the estimate, confidence interval and p-value, based on the output of the subset regression.

(d) (Causal Structure Discovery) Conduct a multiple hypothesis test to identify causal features based on the p-values calculated in step (c).

The consistency of the algorithm for causal structure identification can be established under slightly modified conditions of Theorem 1. To be more precise, we only need to restate the conditions (A1)-(A4) for the joint distribution of $(Y, \boldsymbol{X})$, and then the proof directly follows Theorem 2 of Liang et al. (2015). It is easy to see that the computational complexity of this algorithm is $O\left(n^{3 / 2} p\right)$, as the computational complexity of the SIS algorithm is $O(n p)$ (Fan and Lv, 2008) and there are a total of $O(\sqrt{n} / \log (n))$ features for which the Markov blanket needs to be estimated. Hence, Algorithm 2 can potentially be much faster than the PC-simple and HITON-PC algorithms, especially when $p$ is large. Again, this algorithm can have many implementations. For example, the SIS algorithm (Fan and Lv, 2008) can be used for both variable screening and Markov blanket estimation. The HZ-SIS algorithm (Xue and Liang, 2017) can also be used for both of them.

\subsection{Binary Response}

For binary response data, if we assume that the features $\boldsymbol{X}$ follow a Gaussian distribution, then a joint distribution of $(Y, \boldsymbol{X})$ can be defined as in Lee and Hastie (2015), for which the conditional distribution of each component of $\boldsymbol{X}$ is Gaussian with a linear regression model, and the conditional distribution of $Y$ is a binomial distribution as given by a logistic distribution. Further, we can assume that the joint distribution is faithful to the mixed Graphical model formed by $(Y, \boldsymbol{X})$ (Meek, 1995). As pointed out in Lee and Hastie (2015), the mixed graphical model is a pairwise Markov network and the zero regression coefficients (in the nodewise regression) correspond to the conditional independence. Therefore, Algorithm 2 is also applicable to the binary response 
data, for which variable screening can be done using the GLM SIS algorithm (Fan and Song, 2010). Extending the algorithm to multinomial response data is straightforward. The consistency of the approach directly follows from Theorem 2 of Xu et al. (2019), which shows the consistency of a conditional independence test based approach for learning mixed graphical models. Following Xu et al. (2019), the consistency of the proposed approach can be established under appropriate conditions such as the faithfulness of the joint distribution of $(Y, \boldsymbol{X})$ with respect to the underlying mixed graphical model, the sparsity of Markov blankets, the sparsity of the true model, and some conditions on generalized linear models.

\subsection{Proportional-Hazards}

For Gaussian and binary response data, we justify Algorithm 2 for causal structure discovery by presenting $(Y, \boldsymbol{X})$ as an undirected graph for which the causal structure around $Y$ contains both direct causes and effects of the response variable. Unfortunately, extending this justification to survival data is hard. For survival data, the response variable is proportional hazard, which is non-Gaussian and non-multinomial and thus the joint distribution of $(Y, X)$ is difficult to define with respect to an undirected graph. However, this difficulty can be resolved by modeling $(Y, X)$ as a Bayesian network with $Y$ being a child node only. If $Y$ is a child of $X_{j}$, then $\tilde{\zeta}_{j}=\xi_{j} \cup\{p+1\} \cup \boldsymbol{S}_{*}$ forms the Markov blanket of $X_{j}$, where $\xi_{j}$ is the sub-Markov blanket formed with $\boldsymbol{X}$ as implied by the PC algorithm (Spirtes et al., 2000), $p+1$ is the index of $Y$ (by defining $X_{p+1}=Y$ ), and $\boldsymbol{S}_{*}$ contains all siblings of $X_{j}$ with respect to the common child $Y$. By the total conditioning property shown in Pellet and Elisseeff (2008) for Bayesian networks, we have

$$
X_{j} \perp Y\left|\boldsymbol{X}_{\tilde{\zeta}_{j} \backslash\{j, p+1\}} \Longleftrightarrow X_{j} \perp Y\right| \boldsymbol{X}_{\boldsymbol{V} \backslash\{j, p+1\}},
$$

which implies that Algorithm 2 is still valid for survival data. However, construction of Bayesian networks for non-Gaussian and non-multinomial and with missing data is beyond the scope of this paper. Therefore, there will be no illustrative examples for this part. In (21), if $\tilde{\zeta}_{j}$ is replaced by some super Markov blanket $\tilde{\zeta}_{j}^{\prime} \supset \tilde{\zeta}_{j}$, the equivalence still holds.

This justification is very general and can be applied to the Gaussian and multinomial response data as well. The only shortcoming is that it assumes that $Y$ can only be a child of $\boldsymbol{X}$, while this might be too restrictive for the problems considered with the Gaussian and multinomial response data.

\section{$5 \quad$ Real Data Studies}

This section reports two applications of Algorithm 2, one is for identification of anti-cancer drug sensitive genes, and the other is for identification of cancer driver genes.

\subsection{Identification of Drug Sensitive Genes}

Disease heterogeneity is often observed in complex diseases such as cancer. For example, molecularly targeted cancer drugs are only effective for patients with tumors expressing targets (Grünwald and Hidalgo, 2003; Buzdar, 2009). The disease heterogeneity has directly motivated the development of precision medicine, which aims to improve patient care by tailoring optimal therapies to an individual patient according to his/her molec- 
ular profile and clinical characteristics. Identifying sensitive genes to different drugs is an important step toward the goal of precision medicine.

To illustrate the MNR method, we considered the cancer cell line encyclopedia (CCLE) dataset, which is publicly available at www.broadinstitute.org/ccle. The dataset consists of 8-point dose-response curves for 24 chemical compounds across over 400 cell lines. For different chemical compounds, the numbers of cell lines are slightly different. For each cell line, it consists of the expression values of $p=18,988$ genes. We used the area under the dose-response curve, which was termed as activity area in Barretina et al. (2012), to measure the sensitivity of a drug to each cell line. Compared to other measurements, such as $I C_{50}$ and $E C_{50}$, the activity area could capture the efficacy and potency of the drug simultaneously. An exploratory analysis indicates that treating the activity area as the response of a linear regression with respect to the gene expression values is appropriate.

Since the purpose of this study is to identify the drug sensitive genes instead of assessing the drug effect for all genes, Algorithm 2 was applied with the HZ-SIS algorithm used for variable screening and Markov blanket estimation. In both steps, we set the neighborhood size to be 40 . After getting $p$-values from the subset regressions, the adjusted $p$-values $($ Holm, 1979) were calculated, and the genes with the adjusted $p$-values less than 0.05 were identified as the drug sensitive genes. For some drugs, if there are no genes identified at this significance level, we just selected one gene with the smallest $p$-value. The results were summarized in Table 9 . For Algorithm 2, different neighborhood sizes have been tried, the results are similar.

For comparison, desparsified Lasso, ridge projection and multi sample-splitting were also applied to this example. As in the MNR method, for each drug, we selected the genes with the adjusted $p$-values less than 0.05 as significant; and if there were no genes selected at this significance level, we just reported one gene with the smallest adjusted $p$-value. The results were also summarized in Table 9 .

Compared to the existing methods, MNR performs reasonably well for this real data example. First of all, for all drugs, desparsified Lasso is simply inapplicable due to the ultra-high dimensionality of the dataset; the package $h d i$ aborted due to the excess of memory limit. Due to the same issue, $h d i$ also aborted for some drugs when performing ridge regression. For multi sample-splitting and MNR, it is easy to see that if the same gene is selected by both methods, then the $95 \%$ confidence interval produced by MNR is narrower.

MNR produced promising results in selection of drug sensitive genes. For example, for both drugs Topotecan and Irinotecan, MNR selected the gene SLFN11 as the top drug sensitive gene. In the literature, Barretina et al. (2012) and Zoppoli et al. (2012) reported that SLFN11 is predictive of treatment response for Topotecan and Irinotecan. For drug 17-AAG, MNR selected NQO1 as the top gene; in the literature, Hadley and Hendricks (2014) and Barretina et al. (2012) reported NQO1 as the top predictive biomarker for 17-AAG. For drug Paclitaxel, BNN selected BCL2L1 as the top gene. In the literature, many publications, such as Lee et al. (2016a) and Dorman et al. (2016), reported that the gene BCL2L1 is predictive of treatment response for Paclitaxel. For drug PF2341066, Lawrence and Salgia (2010) reported that HGF, which was selected by MNR as the top drug sensitive gene, is potentially responsible for the effect of PF2341066. For drug LBW242, RIPK1 is selected by MNR. Gaither et al. (2007) and Moriwaki et al. (2015) stated that RIPK1 is one of the presumed target of LBW242, which is involved in increasing death of cells. Finally, we pointed out that the genes selected by MNR have some overlaps with those selected by the multi sample-splitting method, although for the overlapped genes the $95 \%$ confidence intervals produced by MNR tend to be narrower. 
Table 9: Comparison of drug sensitive genes selected by desparsified Lasso, ridge projection, multi samplesplitting (multi-split) and MNR for 24 anti-cancer drugs, where * indicates that this gene was significantly selected and the number in the parentheses denotes the width of the $95 \%$ confidence interval produced by the method.

\begin{tabular}{|c|c|c|c|c|}
\hline Drug & Desparsified Lasso & Ridge & Multi-Split & MNR \\
\hline 17-AAG & - & - & $\mathrm{NQO}^{*}(0.138)$ & $\mathrm{NQO}^{*}(0.115)$ \\
\hline AEW541 & - & F3(0.076) & SP1(0.176) & TMEM229B*(0.142) \\
\hline AZD0530 & - & PPY2(0.966) & SYN3(0.705) & DDAH2(0.088) \\
\hline AZD6244 & - & OSBPL3(0.161) & $\begin{array}{c}\mathrm{SPRY}^{*}(0.084) \\
\mathrm{LYZ}^{*}(0.069) \\
\mathrm{RNF} 125^{*}(0.084)\end{array}$ & $\begin{array}{c}\mathrm{LYZ}^{*}(0.048) \\
\operatorname{SPRY} 2^{*}(0.056)\end{array}$ \\
\hline Erlotinib & - & LRRN1(0.102) & PCDHGC3(0.684) & ENPP1(0.123) \\
\hline Irinotecan & - & SLFN11(0.091) & $\begin{array}{l}\text { ARHGAP19*(0.134) } \\
\text { SLFN11*(0.044) }\end{array}$ & $\begin{array}{c}\text { ARHGAP19*(0.108) } \\
\text { SLFN11*(0.033) }\end{array}$ \\
\hline L-685458 & - & - & MSL2(0.2) & FAM129B(0.187) \\
\hline Lapatinib & - & WDFY4(0.509) & ERBB2* $(0.111)$ & SYTL1(0.062) \\
\hline LBW242 & - & RXFP3(0.86) & LOC100009676(0) & $\operatorname{RIPK1}(0.221)$ \\
\hline Nilotinib & - & - & RAB37(0.187) & $\mathrm{RHOC}(0.103)$ \\
\hline Nutlin-3 & - & TTC7B $(0.119)$ & LOC100009676(0) & DNAJB14(0.163) \\
\hline Paclitaxel & - & $\mathrm{ABCB} 1 *(0.229)$ & $\mathrm{ABCB} 1 *(0.183)$ & BCL2L1*(0.289) \\
\hline Panobinostat & - & C17orf105(1.104) & PUM2(0.589) & TGFB2(0.103) \\
\hline PD-0325901 & - & ZNF646(0.498) & $\begin{array}{c}\mathrm{LYZ}^{*}(0.064) \\
\mathrm{RNF} 125^{*}(0.087)\end{array}$ & DBN1(0.104) \\
\hline PD-0332991 & - & GRM6(0.719) & LOC100506972(0.569) & PUM2(0.244) \\
\hline PF2341066 & - & WDFY4(0.487) & $\mathrm{SPN}^{*}(0.124)$ & $\begin{array}{c}\operatorname{HGF}^{*}(0.043) \\
\operatorname{ENAH}^{*}(0.068) \\
\operatorname{GHRLOS}^{*}(0.24)\end{array}$ \\
\hline PHA-665752 & - & - & LAIR1(0.193) & $\operatorname{INHBB}(0.039)$ \\
\hline PLX4720 & - & ADAMTS13(0.692) & SPRYD5*(0.118) & PLEKHH3(0.22) \\
\hline RAF265 & - & LOC100507235(0.748) & SIGLEC9 $(0.761)$ & SEPT11*(0.078) \\
\hline Sorafenib & - & - & SBNO1(0.426) & $\begin{array}{l}\text { RPL22*(0.151) } \\
\text { LAIR1*(0.094) }\end{array}$ \\
\hline TAE684 & - & - & ARID3A*(0.11) & ARID3A*(0.078) \\
\hline TKI258 & - & - & $\operatorname{SPN}(0.12)$ & KHDRBS1(0.251) \\
\hline Topotecan & - & - & SLFN11*(0.136) & SLFN11*(0.107) \\
\hline ZD-6474 & - & MID1IP1(0.158) & NOD1(0.363) & $\mathrm{PXK}^{*}(0.066)$ \\
\hline
\end{tabular}

\subsection{Identification of Cancer Driver Genes}

We considered the Lymph dataset (Hans et al., 2007), which consists of $n=148$ samples with 100 node-negative cases (low risk for breast cancer) and 48 node-positive cases (high risk for breast cancer) as our binary response. For each sample, there are $p=4512$ genes that showed evidence of variation above the noise level for further 
Table 10: Comparison of the cancer driver genes selected by the MNR, desparsified Lasso and ridge projection methods for the Lymph dataset, where * indicates that this gene was significantly selected.

\begin{tabular}{ccccccc}
\hline & Desparsified Lasso & & Ridge & & \multicolumn{2}{c}{ MNR } \\
\cline { 2 - 3 } \cline { 5 - 6 } \cline { 5 - 6 } Gene & RGS3 & & RGS3 & & RGS3* $^{*}$ & ATP6V1F* \\
95\%C.I. & $(1.145,5.748)$ & & $(-0.251,2.249)$ & & $(0.859,5.178)$ & $(2.073,7.131)$ \\
Width & 4.603 & & 2.500 & & 4.319 & 5.058 \\
\hline
\end{tabular}

study. This dataset has been analyzed by multiple authors, such as Hans et al. (2007) and Liang et al. (2013).

Algorithm 2 was applied to this dataset, where variable screening was done using the GLM SIS algorithm developed in Fan and Song (2010), and the Markov blanket estimation step was done using the HZ-SIS algorithm (Xue and Liang, 2017). In both steps, we set the neighborhood size to be 5. For this dataset, MNR selected two genes, RGS3 and ATP6V1F, with the adjusted p-value less than 0.05. The details were given in Table 10. which are consistent with our existing knowledge. For example, RGS3 is known to play a role in modulating the ability of motile lymphoid cells (Bowman et al., 1998), and to be upregulated in p53-mutated breast cancer tumors (Ooe et al., 2007). ATP6V1F has been reported by many authors in lymph node status studies, see e.g., Hans et al. (2007) and Dobra (2009). For comparison, desparsified Lasso and ridge projection methods were also applied to this example. As aforementioned, the multi sample-splitting algorithm is not yet available for logistic regression. Both desparsified Lasso and ridge projection selected only the gene RGS3 as the cancer driver gene.

A closer look at Table 10 shows that MNR outperforms desparsified Lasso and ridge projection for this example. This can be explained from two perspectives. First, MNR is the only method that identifies RGS3 as a cancer driver gene at an acceptable significance level. While the desparsified Lasso and ridge projection can only identify that RGS3 has a smaller adjusted $p$-value than other genes, and its adjusted $p$-value is greater than 0.05. Second, for the gene RGS3, the $95 \%$ confidence interval produced by MNR is narrower than that produced by desparsified Lasso. Moreover, the $95 \%$ confidence interval produced by ridge projection even contains 0 and is thus less significant.

\section{Discussion}

This paper has proposed the MNR method for constructing confidence intervals and assessing $p$-values for highdimensional regression. The MNR method has successfully broken the high-dimensional inference problem into a series of low-dimensional inference problems based on conditional independence relations among different variables. The embarrassingly parallel structure of the MNR method, where the Markov blanket, confidence interval and $p$-value can be calculated for each variable in parallel, enables it potentially to be run very fast on multicore computers. The MNR method has been tested on high-dimensional linear, logistic and Cox regression. The numerical results indicate that the MNR method significantly outperforms the existing ones. The MNR method has been applied to learn causal structures for high-dimensional linear models with the real data examples for identification of drug sensitive genes and cancer driver genes presented.

This paper has assumed that the features are Gaussian. Extension of the MNR method to non-Gaussian 
features is straightforward. In this case, the conditional independence relations among the features can be figured out using Bayesian networks based on the concept of Markov blanket as described in Section 4.3. The theory developed in Section 2 will still hold. The idea of using conditional independence relations for dimension reduction is general and potentially can be extended to other high-dimensional or big data problems as well.

Finally, we note that the performance of the MNR method relies on the algorithms used for variable selection and Markov blanket estimation, while each of these algorithms can rely on a non-trivial amount of tuning. A sub-optimal performance of these algorithms may adversely affect the performance of the MNR method, for example, the resulting confidence intervals can be wider.

\section{Acknowledgments}

The authors thank the editor, associate editor and two referees for their encouraging and constructive comments which have led to significant improvement of this paper.

\section{Appendix}

\section{A Conditions for Theorem 1}

(A0) The dimension $p_{n}=O\left(\exp \left(n^{\delta}\right)\right)$ for some constant $0 \leq \delta<1 / 2$.

(A1) The distribution $P_{\boldsymbol{X}}$ is multivariate Gaussian, and it satisfies the Markov property and adjacency faithfulness condition with respect to the undirected underlying graph $\boldsymbol{G}$.

(A2) The correlation satisfy $\min \left\{\left|r_{i j}\right| ; e_{i j}=1, i, j=1, \ldots, p_{n}, i \neq j\right\} \geq c_{0} n^{-\kappa}$ for some constants $c_{0}>0$ and $0<\kappa<(1-\delta) / 2$, and $\max \left\{\left|r_{i j}\right| ; i, j=1, \ldots, p_{n}, i \neq j\right\} \leq M_{r}<1$ for some constants $0<M_{r}<1$.

(A3) There exists constants $c_{1}>0,0<\kappa^{\prime} \leq \kappa$, and $0 \leq \tau<1-2 \kappa^{\prime}$ such that $\lambda_{\max }(\Sigma) \leq c_{1} n^{\tau}$.

(A4) The $\psi$-partial correlation coefficients satisfy $\inf \left\{\psi_{i j}: \psi_{i j} \neq 0, i, j=1, \ldots, p_{n}, i \neq j,\left|S_{i j}\right| \leq q_{n}\right\} \geq c_{2} n^{-d}$, where $q_{n}=O\left(n^{2 \kappa^{\prime}+\tau}\right), 0<c_{2}<\infty, 0<d<(1-\delta) / 2$ are some constants, and $S_{i j}$ denotes the conditioning set used in calculating $\psi_{i j}$. In addition, $\sup \left\{\psi_{i j}: i, j=1, \ldots, p_{n}, i \neq j,\left|S_{i j}\right| \leq q_{n}\right\} \geq c_{6} n^{-d} \leq M_{\psi}<1$ for some constants $0<M_{\psi}<1$.

(A5) $\max _{j=1, \ldots, p}\left|\xi_{j}\right|=o\left(n^{1 / 2}\right)$

(A6) There exist constants $c_{3}>0$ and $c_{4}>0$ such that $\min _{j \in \boldsymbol{S}_{*}}\left|\beta_{j}\right| \geq c_{3} n^{-\kappa}$ and $\min _{j \in \boldsymbol{S}_{*}}\left|\operatorname{cov}\left(\beta_{j}^{-1} y, x^{(j)}\right)\right| \geq c_{4}$.

(A7) $\left|\boldsymbol{S}_{*}\right|=o\left(n^{1 / 3}\right)$.

(A8) Other assumptions in Theorem 2 of Fan and Peng (2004).

(A9) Other assumptions in Theorem 1 of Fan et al. (2012) (for the case of random design). 


\section{B Supplementary Materials}

This material is organized as follows. Section B.1 gives the proofs of Lemma 1, Lemma 2 and Theorem 1. Section B.2 presents a variable screening-based MNR method, justifies its validity, and illustrates its performance using a numerical example. Section B.3 presents some figures which illustrate the performance of the MNR method in variable selection.

\section{B.1 Proofs for the Validity of Algorithm 1}

\section{B.1.1 Proof of Lemma 1}

Proof. Without loss of generality, we let $j=1$ and $\{j\} \cup \hat{\xi}_{j} \cup \hat{\boldsymbol{S}}_{*}=\{1, \ldots, d\}=D_{j}$. Let $u^{(i)}=y^{(i)}-\left(\boldsymbol{x}_{D_{j}}^{(i)}\right)^{T} \boldsymbol{\beta}_{D_{j}}^{*}$, then

$$
\sqrt{n}\left(\hat{\boldsymbol{\beta}}_{D_{j}}-\boldsymbol{\beta}_{D_{j}}\right)=\left[\sum_{i=1}^{n} \boldsymbol{x}_{D_{j}}^{(i)}\left(\boldsymbol{x}_{D_{j}}^{(i)}\right)^{T}\right]^{-1}\left[\sqrt{n} \sum_{i=1}^{n} \boldsymbol{x}_{D_{j}}^{(i)} u_{i}\right]=\left[\frac{1}{n} \sum_{i=1}^{n} \boldsymbol{x}_{D_{j}}^{(i)}\left(\boldsymbol{x}_{D_{j}}^{(i)}\right)^{T}\right]^{-1}\left[\frac{1}{\sqrt{n}} \sum_{i=1}^{n} \boldsymbol{x}_{D_{j}}^{(i)} u_{i}\right] .
$$

Since $\boldsymbol{S}_{*} \subseteq \hat{\boldsymbol{S}}_{*} \subseteq\{1, \ldots, d\}$, it's easy to verify $E\left(\boldsymbol{x}_{D_{j}}^{(i)} u_{i}\right)=E_{\boldsymbol{x}_{D_{j}}^{(i)}} E\left(\boldsymbol{x}_{D_{j}}^{(i)} u_{i} \mid \boldsymbol{x}_{D_{j}}^{(i)}\right)=\mathbf{0}$. In addition,

$$
\operatorname{Cov}\left(\boldsymbol{x}_{D_{j}}^{(i)} u_{i}\right)=E_{\boldsymbol{x}_{D_{j}}^{(i)}} \operatorname{Cov}\left(\boldsymbol{x}_{D_{j}}^{(i)} u_{i} \mid \boldsymbol{x}_{D_{j}}^{(i)}\right)+\operatorname{Cov}_{\boldsymbol{x}_{D_{j}}^{(i)}} E\left(\boldsymbol{x}_{D_{j}}^{(i)} u_{i} \mid \boldsymbol{x}_{D_{j}}^{(i)}\right)=\sigma^{2} \Sigma_{d}
$$

where $\Sigma_{d}$ denotes the upper left $d \times d$ submatrix of the covariance matrix $\Sigma($ of $\boldsymbol{X}$ ).

When $\left|D_{j}\right|=d=o\left(n^{1 / 2}\right)$, by Theorem 1.1 of Portnoy (1986),

$$
\frac{1}{\sqrt{n}} \sum_{i=1}^{n} \boldsymbol{x}_{D_{j}}^{(i)} u_{i} \stackrel{d}{\rightarrow} N\left(0, \sigma^{2} \Sigma_{d}\right)
$$

By the weak law of large numbers, $\frac{1}{n} \sum_{i=1}^{n} \boldsymbol{x}_{D_{j}}^{(i)}\left(\boldsymbol{x}_{D_{j}}^{(i)}\right)^{T} \stackrel{p}{\rightarrow} \Sigma_{d}$. Further, by continuous mapping theorem,

$$
\left[\frac{1}{n} \sum_{i=1}^{n} \boldsymbol{x}_{D_{j}}^{(i)}\left(\boldsymbol{x}_{D_{j}}^{(i)}\right)^{T}\right]^{-1} \stackrel{p}{\rightarrow} \Sigma_{d}^{-1}
$$

Combining them together, we have $\sqrt{n}\left(\hat{\boldsymbol{\beta}}_{D_{j}}-\boldsymbol{\beta}_{D_{j}}\right) \stackrel{d}{\rightarrow} N\left(0, \sigma^{2} \Sigma_{d}^{-1}\right)$. Therefore $\sqrt{n}\left(\hat{\beta}_{1}-\beta_{1}\right) \stackrel{d}{\rightarrow} \sigma^{2} \theta_{d, 11}$, where $\theta_{d, 11}$ denotes the $(1,1)$ th entry of the matrix $\Sigma_{d}^{-1}$. As shown around the equations (4) and (5) in the main text, $\theta_{d, 11}=\theta_{11}$, which completes the proof of part (i).

Part (i) implies that $\sqrt{n} \frac{\hat{\beta}_{j}-\beta_{j}}{\sqrt{\sigma^{2} \theta_{j j}}} \sim N(0,1)$ as $n \rightarrow \infty$. Since $\hat{\boldsymbol{S}}_{*} \supseteq \boldsymbol{S}_{*}$ and $\left|D_{j}\right|=o\left(n^{1 / 2}\right), \hat{\sigma}_{n}^{2}$ is a consistent estimator of $\sigma^{2}$. By (IS1),$\hat{\theta}_{11}$ forms a consistent estimator of $\theta_{11}$. Putting all these together, we have $\sqrt{n} \frac{\hat{\beta}_{j}-\beta_{j}}{\sqrt{\hat{\sigma}_{n}^{2}} \hat{\theta}_{j j}} \sim$ $N(0,1)$ as $n \rightarrow \infty$.

\section{B.1.2 Proof of Theorem 1}

Proof. With conditions (A0)-(A4), we can apply Theorem 2 of Liang et al. (2015) to prove that $P\left(\hat{\xi}_{j}=\xi_{j}\right)=$ $1-o(1)$ for all $j=1, \ldots, p_{n}$. Similarly, with conditions (A0), (A3) and (A6)-(A8), we can apply Theorem 5 of Fan and $\mathrm{Lv}(2008)$ to prove that $P\left(\hat{\boldsymbol{S}}_{*}=\boldsymbol{S}_{*}\right)=1-o(1)$. With conditions (A5) and (A7), we have $P\left(\left|D_{j}\right|=\left|\{j\} \cup \hat{\xi}_{j} \cup \hat{\boldsymbol{S}}_{*}\right|=o\left(n^{1 / 2}\right)\right)=1-o(1)$. Following from Lemma 1, we can derive the asymptotic distribution of $\hat{\beta}_{j}$ as expressed in part (i) of Lemma 1. By condition (A9), we can get the consistency of $\hat{\sigma}_{n}^{2}$ based on Theorem 1 of Fan et al. (2012) and the asymptotic $P\left(\left|D_{j}\right| \log \left(p_{n}\right)=o(n)\right)=1-o(1)$. Finally, the proof can be concluded based on Slutsky's theorem. 


\section{B.1.3 Proof of Lemma 2}

Proof. Without loss of generality, we let $j=1$ and $\{j\} \cup \hat{\xi}_{j} \cup \hat{\boldsymbol{S}}_{*}=\{1, \ldots, d\}=D_{j}$. Since $\left|D_{j}\right|=o\left(n^{1 / 2}\right)$ and $\hat{\boldsymbol{S}}_{*} \supseteq \boldsymbol{S}_{*}$ hold, by the theory of GLM estimation with increasing dimensions (Portnoy, 1989), $\sqrt{n}\left(\hat{\beta}_{1}-\beta_{1}\right) \stackrel{d}{\rightarrow}$ $N\left(0, k_{d, 11}\right)$, where $k_{d, 11}$ denotes the $(1,1)$-th entry of the inverse of the Fisher information matrix $I_{d}^{-1}$ for the subset GLM.

In order to show $k_{d, 11}=k_{11}$, we first prove a useful proposition: For any two features $X_{i}$ and $X_{j}$, if at least one of them is not in $\boldsymbol{S}_{*}$, then $k_{i j}=0 \Leftrightarrow \theta_{i j}=0$, where $k_{i j}$ of the $(i, j)$ th entry of $K$ and $\theta_{i j}$ is the $(i, j)$ th entry of the precision matrix $\Theta=\Sigma^{-1}$. Without loss of generality, we assume that $X_{i} \notin \boldsymbol{S}_{*}$. Rewrite the Fisher information matrix $I=E\left(b^{\prime \prime}\left(\boldsymbol{x}^{T} \boldsymbol{\beta}\right) \boldsymbol{x} \boldsymbol{x}^{T}\right)$ as $E\left(\boldsymbol{z} \boldsymbol{z}^{T}\right)$, where $\boldsymbol{z}=\sqrt{b^{\prime \prime}\left(\boldsymbol{x}^{T} \boldsymbol{\beta}\right)} \boldsymbol{x}=\sqrt{b^{\prime \prime}\left(\boldsymbol{x}_{\boldsymbol{S}_{*}}^{T} \boldsymbol{\beta}_{\boldsymbol{S}_{*}}\right)} \boldsymbol{x}$. Therefore, $K=I^{-1}$ measures the partial correlation structure of $\boldsymbol{z}$, and $k_{i j}=0$ if and only if the partial correlation of $\boldsymbol{z}_{i}$ and $\boldsymbol{z}_{j}$ is zero. Further, recall that the partial correlation of $\boldsymbol{z}_{i}$ and $\boldsymbol{z}_{j}$ equals zero if and only if the coefficient $\tilde{c}_{i j}$ in the nodewise regression $\boldsymbol{z}_{i}=\sum_{t \neq i} \tilde{c}_{i t} \boldsymbol{z}_{t}$ equals zero, where $\left\{\tilde{c}_{i t}: t \neq i\right\}$ satisfies

$$
\left\{\tilde{c}_{i t}: t \neq i\right\}=\arg \min _{\left\{c_{i t}: t \neq i\right\}} E\left[\boldsymbol{z}_{i}-\sum_{t \neq i} c_{i t} \boldsymbol{z}_{t}\right]^{2} .
$$

Note that for any set of Gaussian variables $\left\{\boldsymbol{x}_{1}, \ldots, \boldsymbol{x}_{p_{n}}\right\}$,

$$
\left\{\tilde{c}_{i t}^{\prime}: t \neq i\right\}=\arg \min _{\left\{c_{i t}^{\prime}: t \neq i\right\}} E\left[\boldsymbol{x}_{i}-\sum_{t \neq i} c_{i t}^{\prime} \boldsymbol{x}_{t}\right]^{2}=\arg \min _{\left\{c_{i t}^{\prime}: t \neq i\right\}} E\left\{\left[\boldsymbol{x}_{i}-\sum_{t \neq i} c_{i t}^{\prime} \boldsymbol{x}_{t}\right]^{2} \mid\left\{\boldsymbol{x}_{1}, \ldots, \boldsymbol{x}_{p_{n}}\right\} \backslash \boldsymbol{x}_{i}\right\},
$$

as $E\left[\boldsymbol{x}_{i}-\sum_{t \neq i} c_{i t}^{\prime} \boldsymbol{x}_{t}\right]^{2}$ is minimized if and only if $\sum_{t \neq i} c_{i t}^{\prime} \boldsymbol{x}_{t}=E\left[\boldsymbol{x}_{i} \mid\left\{\boldsymbol{x}_{1}, \ldots, \boldsymbol{x}_{p_{n}}\right\} \backslash \boldsymbol{x}_{i}\right]$.

Since we have assumed $\boldsymbol{S}_{*} \subseteq\{t: t \neq i\}$, it is easy to verify that

$$
\begin{aligned}
& \arg \min _{\left\{c_{i t}: t \neq i\right\}} E\left\{\left[\boldsymbol{z}_{i}-\sum_{t \neq i} c_{i t} \boldsymbol{z}_{t}\right]^{2} \mid\left\{\boldsymbol{z}_{1}, \ldots, \boldsymbol{z}_{p_{n}}\right\} \backslash \boldsymbol{z}_{i}\right\} \\
= & \arg \min _{\left\{c_{i t}: t \neq i\right\}} E\left\{\left[\sqrt{b^{\prime \prime}\left(\boldsymbol{x}_{\boldsymbol{S}_{*}}^{T} \boldsymbol{\beta}_{\boldsymbol{S}_{*}}\right)} \boldsymbol{x}_{i}-\sum_{t \neq i} c_{i t} \sqrt{\left.b^{\prime \prime}\left(\boldsymbol{x}_{\boldsymbol{S}_{*}}^{T} \boldsymbol{\beta}_{\left.\boldsymbol{S}_{*}\right)} \boldsymbol{x}_{t}\right]^{2} \mid\left\{\boldsymbol{x}_{1}, \ldots, \boldsymbol{x}_{p_{n}}\right\} \backslash \boldsymbol{x}_{i}\right\}}\right.\right. \\
= & \sqrt{b^{\prime \prime}\left(\boldsymbol{x}_{\boldsymbol{S}_{*}}^{T} \boldsymbol{\beta}_{\boldsymbol{S}_{*}}\right)} \arg \min _{\left\{c_{i t}: t \neq i\right\}} E\left\{\left[\boldsymbol{x}_{i}-\sum_{t \neq i} c_{i t} \boldsymbol{x}_{t}\right]^{2} \mid\left\{\boldsymbol{x}_{1}, \ldots, \boldsymbol{x}_{p_{n}}\right\} \backslash \boldsymbol{x}_{i}\right\}=\left\{\tilde{c}_{i t}^{\prime}: t \neq i\right\} .
\end{aligned}
$$

Since this holds for any fixed $\left\{\boldsymbol{z}_{1}, \ldots, \boldsymbol{z}_{p_{n}}\right\} \backslash \boldsymbol{z}_{i}$, we further have

$$
\left\{\tilde{c}_{i t}: t \neq i\right\}=\arg \min _{\left\{c_{i t}: t \neq i\right\}} E\left[\boldsymbol{z}_{i}-\sum_{t \neq i} c_{i t} \boldsymbol{z}_{t}\right]^{2}=\arg \min _{\left\{c_{i t}: t \neq i\right\}} E\left\{\left[\boldsymbol{z}_{i}-\sum_{t \neq i} c_{i t} \boldsymbol{z}_{t}\right]^{2} \mid\left\{\boldsymbol{z}_{1}, \ldots, \boldsymbol{z}_{p_{n}}\right\} \backslash \boldsymbol{z}_{i}\right\}=\left\{\tilde{c}^{\prime}{ }_{i t}: t \neq i\right\} .
$$

Putting these together, we have $k_{i j}=0 \Leftrightarrow \tilde{c}_{i j}=0 \Leftrightarrow \tilde{c}^{\prime}{ }_{i j}=0 \Leftrightarrow \theta_{i j}=0$, which justifies the proposition.

Next, we partition $K$ as

$$
K=\left[\begin{array}{cc}
K_{d} & K_{d, p-d} \\
K_{p-d, d} & K_{p-d}
\end{array}\right]
$$

By the formula of partitioned matrix inverse, we have $I_{d}=\left(K_{d}-K_{d, p-d} K_{p-d}^{-1} K_{p-d, d}\right)^{-1}$ and $I_{d}^{-1}=K_{d}-$ $K_{d, p-d} K_{p-d}^{-1} K_{p-d, d}$. Since the true features set $\boldsymbol{S}_{*}$ has been included in $\{1, \ldots, d\}$, we can apply the previous proposition to obtain that $k_{1 j}=0 \Leftrightarrow \theta_{1 j}=0$ for $j>d$. Since the Markov neighborhood of $\boldsymbol{x}_{1}$ has been included in $\{1, \ldots, d\}$, we have $\theta_{1 j}=0$ for $j>q$. Combining these two together, we have $k_{1 j}=0$ for $j>q$; in other 
words, the first row of $K_{d, p-d}$ and first column of $K_{p-d, d}$ are exactly zero. Therefore, the $(1,1)$-th element of $K_{d, p-d} K_{p-d}^{-1} K_{p-d, d}$ is also exactly zero, and the (1,1)-th entry of $K_{d}$ equals to the $(1,1)$-th entry of $I_{d}^{-1}$, i.e., $k_{11}=k_{d, 11}$. This completes the proof of part (i).

Since for the GLMs, including the logistic, Poisson and Cox regression, $J_{n}(\boldsymbol{\beta})$ is almost surely Lipschitz continuous, $J_{n}(\boldsymbol{\beta})$ is stochastically equicontinuous. Therefore,

$$
\left|J_{n}\left(\hat{\boldsymbol{\beta}}_{D_{j}}\right)-I\left(\boldsymbol{\beta}_{D_{j}}\right)\right| \leq\left|J_{n}\left(\hat{\boldsymbol{\beta}}_{D_{j}}\right)-J_{n}\left(\boldsymbol{\beta}_{D_{j}}\right)\right|+\left|J_{n}\left(\boldsymbol{\beta}_{D_{j}}\right)-I\left(\boldsymbol{\beta}_{D_{j}}\right)\right| \stackrel{p}{\rightarrow} 0
$$

where $\left|J_{n}\left(\hat{\boldsymbol{\beta}}_{D_{j}}\right)-J_{n}\left(\boldsymbol{\beta}_{D_{j}}\right)\right| \stackrel{p}{\rightarrow} 0$ follows from the stochastic equicontinuity of $J_{n}(\cdot)$ and the consistency of the $\operatorname{MLE} \hat{\boldsymbol{\beta}}_{D_{j}}$, and $\left|J_{n}\left(\boldsymbol{\beta}_{D_{j}}\right)-I\left(\boldsymbol{\beta}_{D_{j}}\right)\right| \stackrel{p}{\rightarrow} 0$ follows from the weak law of large numbers. This completes the proof of part (ii).

\section{B.2 A Variable Screening-Based MNR Algorithm}

\section{B.2.1 The Algorithm}

Algorithm S1. (Variable screening-based Markov neighborhood regression)

(a) (Variable screening) Apply the sure independence screening procedure (Fan and Lv, 2008) to obtain a reduced feature set, $\hat{\boldsymbol{S}}_{*} \subseteq\{1, \ldots, p\}$, with the size $\left|\hat{\boldsymbol{S}}_{*}\right|=O(\sqrt{n} / \log (n))$.

(b) (Markov blanket estimation) Apply the correlation screening procedure (Luo et al., 2014) to $\boldsymbol{X}$ to obtain a reduced neighborhood $\hat{\xi}_{j} \subseteq\{1, \ldots, p\}$ for each variable $X_{j}$ with the size $\left|\hat{\xi}_{j}\right|=O(\sqrt{n} / \log (n))$.

(c) (Subset Regression) For each variable $X_{j}, j=1, \ldots, p$, run the OLS regression with the features given by $\left\{X_{j}\right\} \cup \boldsymbol{X}_{\hat{\xi}_{j}} \cup \boldsymbol{X}_{\hat{\boldsymbol{S}}_{*}}$. Conduct inference for $\beta_{j}$, including the estimate, confidence interval and $p$-value, based on the output of the subset regression.

The variable screening step restricts the size of $\hat{\boldsymbol{S}}_{*}$ to $O(\sqrt{n} / \log (n))$, which looks more restrictive than the order $O(n / \log (n))$ used by conventional variable screening algorithms. However, this is just a technical condition and will not affect much on the the actual size of $\hat{\boldsymbol{S}}_{*}$. For the step of Markov blanket estimation, this is similar. To justify the validity of Algorithm [S1, we establish Theorem S1 with part of the conditions given as follows:

(B1) The distribution $P_{\boldsymbol{X}}$ is multivariate Gaussian, and it satisfies the Markov property with respect to the undirected graph $\boldsymbol{G}$ for all size of $\boldsymbol{X}$.

(B2) The correlation coefficients satisfy $\min \left\{\left|r_{i j}\right| ; e_{i j}=1, i, j=1, \ldots, p_{n}, i \neq j\right\} \geq c_{0} n^{-\kappa}$ for some constants $c_{0}>0$ and $0<\kappa<1 / 4$, and $\max \left\{\left|r_{i j}\right| ; i, j=1, \ldots, p_{n}, i \neq j\right\} \leq M_{r}<1$ for some constants $0<M_{r}<1$.

(B3) There exists constants $c_{1}>0$ and $0 \leq \tau<\frac{1}{2}-2 \kappa$ such that $\lambda_{\max }(\Sigma) \leq c_{1} n^{\tau}$.

Theorem S1. (Validity of Algorithm S1) If the conditions (A0), (A6), (A9) (given in the Appendix of the main text), and (B1)-(B3) hold, the sure independence screening (SIS) algorithm (Fan and Lv, 2008) is used for variable selection in step (a), and the correlation screening algorithm (Luo et al., 2014) is used for estimating $\xi_{j}$ 's in step (b), then for each $j \in\left\{1,2, \ldots, p_{n}\right\}, \sqrt{n} \frac{\hat{\beta}_{j}-\beta_{j}}{\sqrt{\hat{\sigma}_{n}^{2} \hat{\theta}_{j j}}} \sim N(0,1)$ as $n \rightarrow \infty$, where $\hat{\beta}_{j}$ denotes the estimate 
of $\beta_{j}$ obtained from the subset regression, $\hat{\sigma}_{n}^{2}=\left(\boldsymbol{y}-\boldsymbol{x}_{D_{j}} \hat{\boldsymbol{\beta}}_{D_{j}}\right)^{T}\left(\boldsymbol{y}-\boldsymbol{x}_{D_{j}} \hat{\boldsymbol{\beta}}_{D_{j}}\right) /(n-d-1), \hat{\theta}_{j j}$ is the $(j, j)-t h$ entry of the matrix $\left[\frac{1}{n} \sum_{i=1}^{n} \boldsymbol{x}_{D_{j}}^{(i)}\left(\boldsymbol{x}_{D_{j}}^{(i)}\right)^{T}\right]^{-1}$, and $\boldsymbol{x}_{D_{j}}^{(i)}$ denotes the $i$-th row of $\boldsymbol{X}_{D_{j}}$.

Proof. With conditions (A0) and (B1)-(B3), we can apply Theorem 1 and Theorem 2 of Luo et al. (2014) to obtain that $P\left(\hat{\xi}_{j} \supseteq \xi_{j}\right)=1-o(1)$, for $j=1, \ldots, p_{n}$. In addition, $P\left(\left|\hat{\xi}_{j}\right| \leq o\left(n^{\frac{1}{2}}\right)\right)=1-o(1)$ holds with an appropriate threshold of correlation coefficients. Similarly, with the conditions (A0), (A6), and (B3), we can apply Theorem 1 of Fan and Lv (2008) to obtain that $P\left(\hat{\boldsymbol{S}}_{*} \supseteq \boldsymbol{S}_{*}\right)=1-o(1)$. According to Theorem 1 of Fan and Lv (2008), the size of $\hat{\boldsymbol{S}}_{*}$ can be chosen in the order of $O\left(n^{1-\vartheta}\right)$ for some $\vartheta<1-2 \kappa-\tau$, i.e., $1-\vartheta>2 \kappa+\tau$. While, by (B3), we have $2 \kappa+\tau=\frac{1}{2}-\varepsilon<1 / 2$ for some $\varepsilon>0$. Therefore, we can choose $1-\vartheta=\frac{1}{2}-\frac{\varepsilon}{2}$, which implies $\left|\hat{\boldsymbol{S}}_{*}\right|=o\left(n^{1 / 2}\right)$. As suggested in the algorithm, we can choose the size of $\hat{\boldsymbol{S}}_{*}$ in the order $O(\sqrt{n} / \log (n))$. Putting these together, we have $P\left(\left|D_{j}\right|=\left|\{j\} \cup \hat{\xi}_{j} \cup \hat{\boldsymbol{S}}_{*}\right|=o\left(n^{1 / 2}\right)\right)=1-o(1)$. By condition (A9), we can get the consistency of $\hat{\sigma}_{n}^{2}$ based on Theorem 1 of Fan et al. (2012) and the asymptotic $P\left(\left|D_{j}\right| \log \left(p_{n}\right)=o(n)\right)=1-o(1)$. Finally, by applying Lemma 1 and Slutsky's theorem, we can conclude the proof of the theorem.

Algorithm S1 can have many variants. For example, we can replace step (a) by Lasso, which has been empirically found to perform rather well for screening in comparison to marginal correlation screening, see Bühlmann and Mandozzi (2014) for empirical comparisons. We can also replace step (a) by the Henze-Zirkler sure independence screening (HZ-SIS) algorithm (Xue and Liang, 2017).

HZ-SIS is a model-free algorithm, which is developed based on Henze-Zirkler's multivariate normality test (Henze and Zirkler, 1990). When applying HZ-SIS to Gaussian data, the nonparanormal transformation (Liu et al., 2009) step might be omitted, but including such a step, which provides an appropriate truncation for extreme values, might robustify the performance of the algorithm. For Gaussian data, this algorithm is equivalent to test whether the correlation coefficient is zero. Since HZ-SIS possesses the ranking consistency property, which stems from the consistency of the Henze-Zirkler's test and is established in Theorem S2 below, it is expected to perform better than the SIS algorithm by Fan and Lv (2008). The ranking consistency property is a stronger property than sure screening, which enables an asymptotic separation of the cases of zero correlation and non-zero correlation. Further, under condition (C4), we will be able to restrict the size of $\hat{\boldsymbol{S}}_{*}$ to $O(\sqrt{n} / \log (n))$.

Ranking Consistency of the HZ-SIS Algorithm: Let $D=\min \left\{k: Y\right.$ and $X_{k}$ are marginally dependent $\}$, and let $I=\min \left\{k: Y\right.$ and $X_{k}$ are marginally independent $\}$. Let $\omega_{k}$ denote the screening statistic for the variable $X_{k}$, which is defined by

$$
\omega_{k}=\int_{\mathbb{R}^{2}}\left|\psi_{k}(\boldsymbol{t})-\exp \left(-\frac{1}{2} \boldsymbol{t}^{\prime} \boldsymbol{t}\right)\right| \phi_{\beta}(\boldsymbol{t}) d \boldsymbol{t}
$$

where $\phi_{\beta}(\boldsymbol{t})$ is the PDF of $N\left(0, \beta^{2} I_{2}\right), \beta$ is a smooth parameter with the optimal value $(1.25 n)^{1 / 6} / \sqrt{2}$; and $\psi_{k}(\boldsymbol{t})$ is the characteristic function of $\left(\Phi^{-1}\left(F_{k}\left(X_{k}\right)\right), \Phi^{-1}\left(F_{y}(Y)\right)\right), \Phi$ denotes the CDF of the standard normal distribution, and $F_{k}$ and $F_{y}$ denote the CDFs of $X_{k}$ and $Y$, respectively.

(C1) There exist positive constants $c>0$ and $0 \leq \kappa \leq 1 / 4$ such that $\min _{k \in D} \omega_{k} \geq 2 c n^{-\kappa}$.

(C2) The dimension $p=O\left(\exp \left(n^{\tau}\right)\right)$ for some constant $0 \leq \tau<(1-4 \kappa) / 3$. 
(C3) $\liminf \operatorname{in}_{p \rightarrow \infty}\left\{\min _{k \in D} \omega_{k}-\max _{k \in I} \omega_{k}\right\} \geq c_{3}$, where $c_{3}>0$ is a constant.

(C4) $|D|=o(\sqrt{n})$.

Since the Henze-Zirkler test is consistent (Henze and Zirkler, 1990), for which the power tends to 1 for all cases in $D,(\mathrm{C} 3)$ is a relatively weaker assumption. Let $\hat{\omega}_{k}$ denote the Henze-Zirkler test statistic, i.e., an estimator of $\omega_{k}$, calculated with $\beta=(1.25 n)^{1 / 6} / \sqrt{2}$ and $n$ observations, and let $\hat{D}_{n}$ denote the estimate of $D$. Under conditions $(\mathrm{C} 1)$ and $(\mathrm{C} 2)$, Xue and Liang (2017) proved the screening property $P(D \subseteq \hat{D})=1-o(1)$ as $n \rightarrow \infty$. Under conditions (C1)-(C3), the ranking consistency property can be established as follows:

Theorem S2. If conditions (C1)-(C3) hold, then $\liminf _{n \rightarrow \infty}\left\{\min _{k \in D} \hat{\omega}_{k}-\max _{k \in I} \hat{\omega}_{k}\right\}>0$, a.s.

Proof. The theorem can be proved in a similar way to Theorem 2.2 of Cui et al. (2015). Calculate the probability

$$
\begin{aligned}
& P\left\{\min _{k \in D} \hat{\omega}_{k}-\max _{k \in I} \hat{\omega}_{k}<c_{3} / 2\right\} \leq P\left\{\left(\min _{k \in D} \hat{\omega}_{k}-\max _{k \in I} \hat{\omega}_{k}\right)-\left(\min _{k \in D} \omega_{k}-\max _{k \in I} \omega_{k}\right)<-c_{3} / 2\right\} \\
& \leq P\left\{\left|\left(\min _{k \in D} \hat{\omega}_{k}-\max _{k \in I} \hat{\omega}_{k}\right)-\left(\min _{k \in D} \omega_{k}-\max _{k \in I} \omega_{k}\right)\right|>c_{3} / 2\right\} \leq P\left\{2 \max _{1 \leq k \leq p}\left|\hat{\omega}_{k}-\omega_{k}\right|>c_{3} / 2\right\} \\
& \leq O\left(p \exp \left(-c_{1} n^{\frac{1-4 k}{3}}\right)\right),
\end{aligned}
$$

by letting $c_{3}=n^{-\kappa}$, where the last inequality follows from Lemma 1 of Xue and Liang (2017). Then, for some $n_{0}, \sum_{n=n_{0}}^{\infty} p \exp \left(-c_{1} n^{\frac{1-4 \kappa}{3}}\right) \leq \sum_{n=n_{0}}^{\infty} n^{-2}<\infty$. Therefore, we obtain that $\liminf _{n \rightarrow \infty}\left\{\min _{k \in D} \hat{\omega}_{k}-\right.$ $\left.\max _{k \in I} \hat{\omega}_{k}\right\} \geq c_{3} / 2>0$ a.s.

\section{B.2.2 Illustration of Algorithm $\underline{\text { S1 }}$}

Algorithm S1 was also applied to the same 100 datasets simulated in Section 3.2 of the main text. For simplicity, we set the same upper bound for the Markov blanket size $\left|\hat{\xi}_{j}\right|$ and the model size $\left|\hat{\boldsymbol{S}}_{*}\right|$. Let $m$ denote the upper bound. Table S1 summarizes the outputs of the algorithm with different values of $m=3,5,8,15$ and 20 . The results indicate that Algorithm $\mathbf{S 1}$ performs well for this example. Although the widths of the resulting confidence intervals vary with the value of $m$, the coverage rates are pretty stable, which are always close to the nominal level 0.95 for both zero and nonzero regression coefficients. In the case $m=3$, where some true features are missed in each subset regression, Algorithm [\$1 still performs reasonably well, although the resulting confidence intervals are a little wider. When $m=8$, the algorithm attains its best performance. Of course, such a choice of $m$ depends on how the data was generated. Intuitively, the best choice of $m$ should be the smallest size such that all true features can be covered by a reduced feature set of that size and for almost every variable, its Markov blanket can be covered by a reduced neighborhood of that size.

A comparison of Table 1 (of the main text) and Table S1 shows that the confidence intervals produced by Algorithm [1] tend to be wider than those by Algorithm 1. This is reasonable, as Algorithm 1 seeks to use the smallest Markov neighborhood for each feature and thus the resulting inference is more accurate. However, as shown in Table [2, Algorithm [1] is much more efficient than Algorithm 1 in computation. Algorithm [1] is an accuracy/efficiency trade-off version of Algorithm 1.

\section{B.2.3 Computational Complexity}

In Algorithm S1, SIS was used in both variable screening and Markov blanket estimation steps. By Fan and Lv (2008), the computational complexity of the variable screening step is $O(n p)$. Therefore, the computational 
Table S1: Coverage rates and widths of the 95\% confidence intervals produced by Algorithm S1 for the Toeplitzcovariance linear regression with different values of $m$, which controls the size of Markov neighborhoods. Refer to the caption of Table 1 of the main text for the notation.

\begin{tabular}{ccccccc}
\hline Measure & & $m=3$ & $m=5$ & $m=8$ & $m=15$ & $m=20$ \\
\hline \multirow{2}{*}{ Coverage } & signal & $0.956(0.021)$ & $0.962(0.019)$ & $0.956(0.021)$ & $0.958(0.020)$ & $0.954(0.021)$ \\
& noise & $0.952(0.021)$ & $0.950(0.022)$ & $0.951(0.022)$ & $0.951(0.022)$ & $0.949(0.022)$ \\
\hline \multirow{2}{*}{ Width } & signal & $1.023(0.035)$ & $0.854(0.014)$ & $0.839(0.011)$ & $0.857(0.011)$ & $0.876(0.011)$ \\
& noise & $2.566(0.019)$ & $1.610(0.054)$ & $0.902(0.008)$ & $0.935(0.007)$ & $0.963(0.008)$ \\
\hline
\end{tabular}

complexity of the Markov blanket estimation step is $O\left(n p^{2}\right)$, as SIS needs to be done for each of $p$ features. By the size of the Markov neighborhood, the computational complexity of the subset regression step is $O\left(n^{2} p\right)$. Therefore, the total computational complexity of Algorithm S1 is $O\left(n p^{2}\right)$ for small-n-large- $p$ problems. In contrast, as analyzed in Section 3.5 of the main text, the computational complexity of Algorithm 1 is $O\left(n^{3} p^{2}\right)$.

For the dataset used in Section 3.5 of the main text, Algorithm S1 cost only 3.9 seconds when running with a single thread on an Intel(R) Xeon(R) CPU E5-2660 v3@2.60GHz machine. Table $\$ 2$ compares the CPU time cost by different methods for the same dataset on the same machine, where the parts for desparsified-Lasso and Algorithm 1 are taken from Table 8 of the main text.

Table S2: CPU times (in seconds) cost by different methods for a dataset with $n=200$ and $p=500$, where $\mathrm{MNR}_{a}, \mathrm{MNR}_{b}, \mathrm{MNR}_{c}$, and $\mathrm{MNR}_{d}$ represent different implementations of Algorithm 1 as explained in Table 8 of the main text.

\begin{tabular}{ccccccc}
\hline & & \multicolumn{4}{c}{ Algorithm 1 } & \\
\cline { 3 - 5 } Methods & Desparsified-Lasso & $\mathrm{MNR}_{a}$ & $\mathrm{MNR}_{b}$ & $\mathrm{MNR}_{c}$ & $\mathrm{MNR}_{d}$ & Algorithm S1 \\
\hline CPU(s) & 258 & 152 & 230 & 205 & 250 & 3.9 \\
\hline
\end{tabular}

\section{B.3 Figures Illustrating the Performance of MNR in Variable Selection}




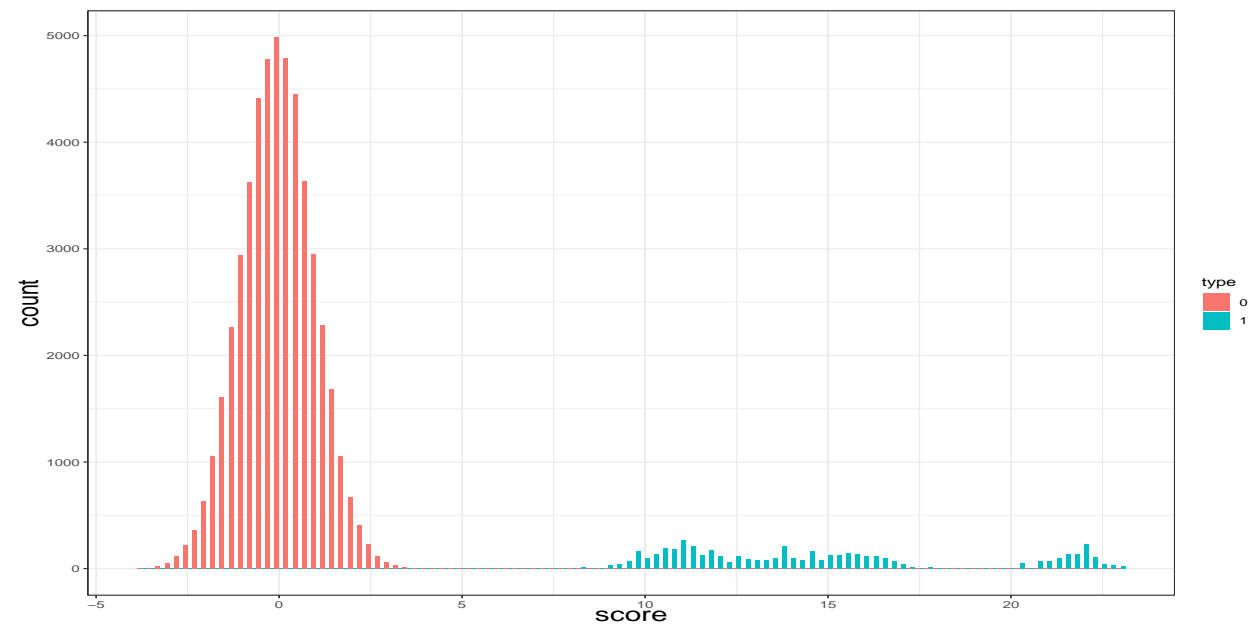

Figure S1: Histogram of the z-scores produced by MNR for the Toeplitz-covariance linear regression example, where the z-scores of the true and false features are shown in blue and red, respectively. The z-scores of the true features have been replicated 10 times for a better view of the plot.

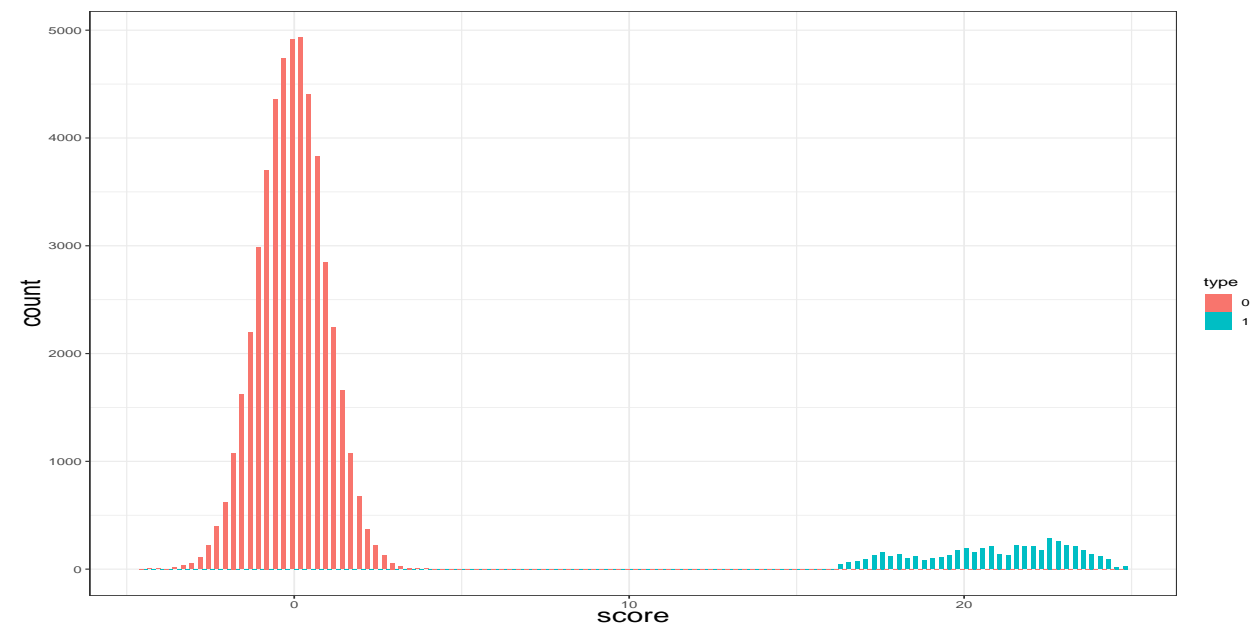

Figure S2: Histogram of the z-scores produced by MNR for the AR(2)-precision linear regression example, where the z-scores of the true and false features are shown in blue and red, respectively. The z-scores of the true features have been replicated 10 times for a better view of the plot. 


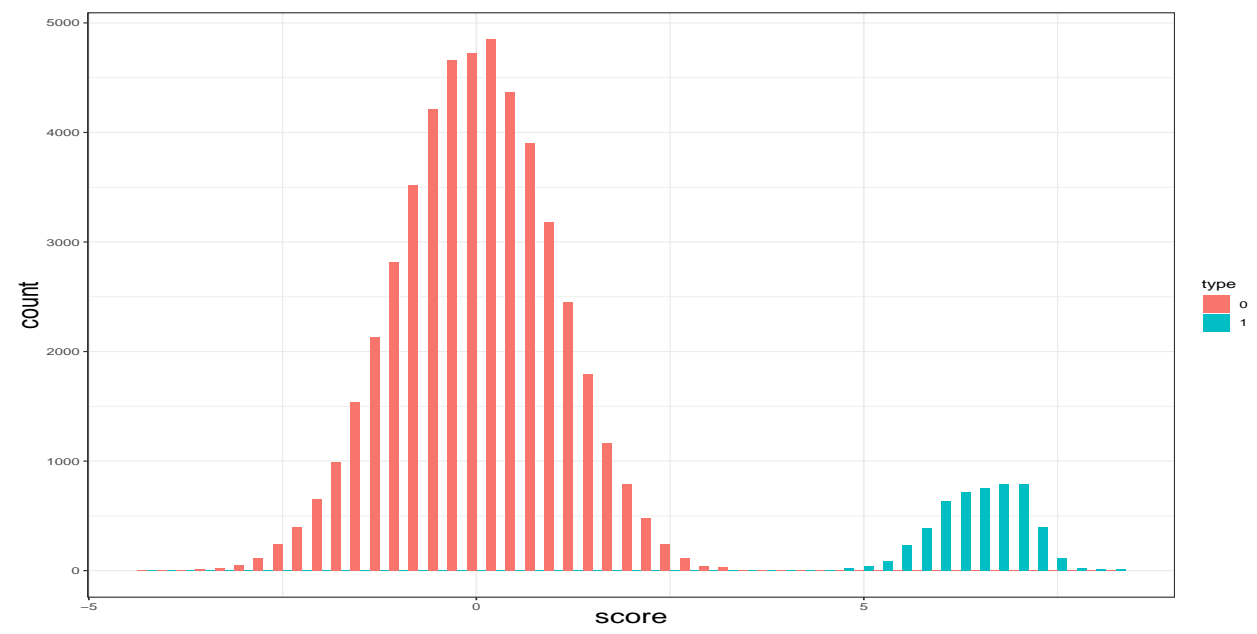

Figure S3: Histogram of the z-scores produced by MNR for the AR(2)-precision logistic regression example, where the z-scores of the true and false features are shown in blue and red, respectively. The z-scores of the true features have been replicated 10 times for a better view of the plot.

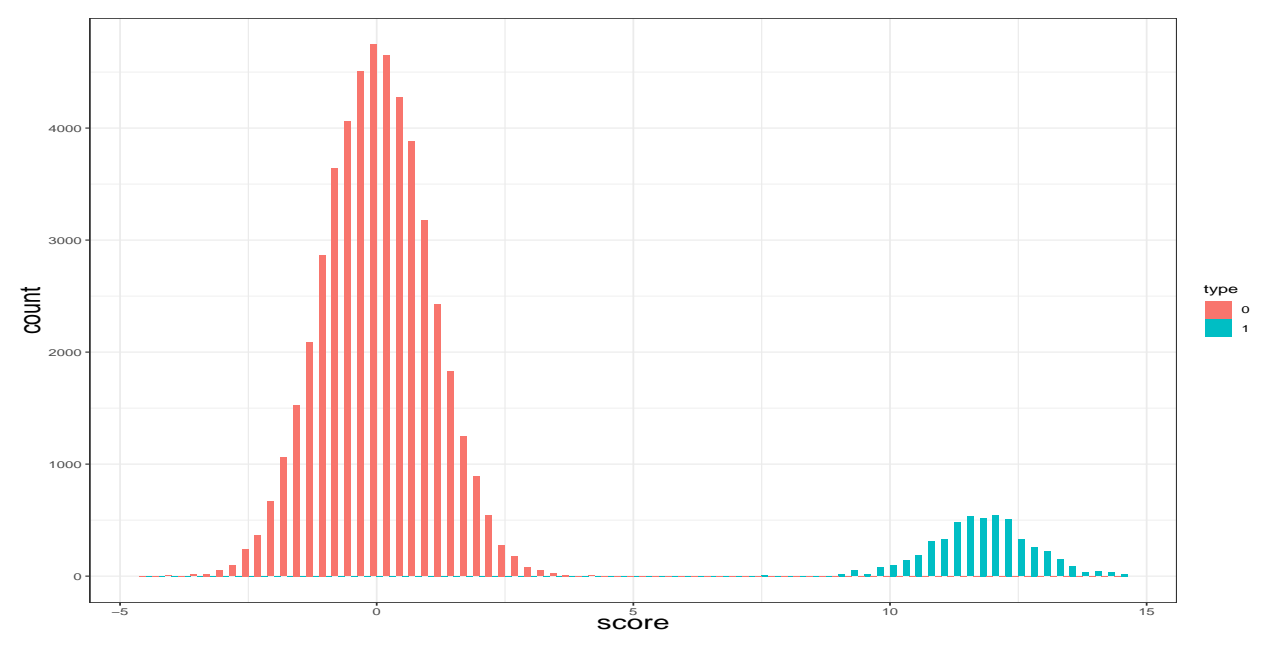

Figure S4: Histogram of the z-scores produced by MNR for the AR(2)-precision Cox regression example, where the z-scores of the true and false features are shown in blue and red, respectively. The z-scores of the true features have been replicated 10 times for a better view of the plot. 


\section{References}

Aliferis, C., Statnikov, A., Tsamardinos, I., Mani, S., and Koutsoukos, X. D. (2010), "Local causal and Markov blanket induction for causal discovery and feature selection for classification, Part I: Algorithms and empirical evaluation," Journal of Machine Learning Research, 11, 171-234.

Barretina, J., Caponigro, G., Stransky, N., Venkatesan, K., Margolin, A. A., Kim, S., Wilson, C. J., Lehár, J., Kryukov, G. V., Sonkin, D., Reddy, A., Liu, M., Murray, L., Berger, M. F., Monahan, J. E., Morais, P., Meltzer, J., Korejwa, A., Jané-Valbuena, J., Mapa, F. A., Thibault, J., Bric-Furlong, E., Raman, P., Shipwayn, A., Engels, I. H., Cheng, J., Yu, G. K., Yu, J., Aspesi, P., de Silva, M., Jagtap, K., Jones, M. D., Wang, L., Hatton, C., Palescandolo, E., Gupta, S., Mahan, S., Sougnez, C., Onofrio, R. C., Liefeld, T., MacConaill, L., Winckler, W., Reich, M., Li, N., Mesirov, J. P., Gabriel, S. B., Getz, G., Ardlie, K., Chan, V., Myer, V. E., Weber, B. L., Porter, J., Warmuth, M., Finan, P., Harris, J. L., Meyerson, M., Golub, T. R., Morrissey, M. P., Sellers, W. R., Schlegel, R., and Garraway, L. A. (2012), "The Cancer cell line encyclopedia enables predictive modeling of anticancer drug sensitivity," Nature, 483, 603-607.

Belloni, A., Chernozhukov, V., and Kato, K. (2015), "Uniform post-selection inference for least absolute deviation regression and other Z-estimation problems," Biometrika, 102, 77-94.

Berk, R., Brown, L., Buja, A., Zhang, K., and Zhao, L. (2013), "Valid post-selection inference," Annals of Statistics, 41, 802-837.

Bowman, E., Campbell, J., Druey, K., Scheschonka, A., Kehrl, J., and Butcher, E. (1998), "Regulation of chemotactic and proadhesive responses to chemoattractant receptors by RGS (regulator of G-protein signaling) family members," Journal of Biological Chemistry, 273, 28040-28048.

Bühlmann, P. (2013), "Statistical significance in high-dimensional linear models," Bernoulli, 19, 1212-1242.

Bühlmann, P., Kalisch, M., and Maathuis, M. (2010), "Variable selection in high-dimensional linear models: patially faithful distributions and the PC-simple algorithm," Biometrika, 97, 261-278.

Bühlmann, P. and Mandozzi, J. (2014), "High-dimensional variable screening and bias in subsequent inference, with an empirical comparison," Comput. Statist., 29, 407-430.

Bühlmann, P. and van de Geer, S. (2011), Statistics for High-Dimensional Data: Methods, Theory and Applications, New York: Springer.

Buzdar, A. (2009), "Role of biologic therapy and chemotherapy in hormone receptor and HER2-positive breast cancer," The Annals of Oncology, 20, 993-999.

Chatterjee, A. and Lahiri, S. (2013), "Rates of convergence of the adaptive LASSO estimators to the oracle distribution and higher order refinements by the bootstrap," Annals of Statistics, 41, 1232-1259.

Cox, D. (1975), "Partial Likelihood," Biometrika, 62, 269-276.

Cui, H., Li, R., and Zhong, W. (2015), "Model-free feature screening for ultrahigh dimensional discriminant analysis," Journal of the American Statistical Association, 110, 630-641. 
Dezeure, R., Bühlmann, P., Meier, L., and Meinshausen, N. (2015), "High-dimensional inference: Confidence intervals, $p$-values and R-software hdi," Statistical Science, 30, 533-558.

Dobra, A. (2009), "Variable selection and dependency networks for genomewide data," Biostatistics, 10, 621639.

Dorman, S., Baranova, K., Knoll, J., Urquhart, B., Mariani, G., Carcangiu, M., and Rogan, P. (2016), "Genomic signatures for paclitaxel and gemcitabine resistance in breast cancer drived by machine learning," Molecular Oncology, 10, 85-100.

Fan, J., Guo, S., and Hao, N. (2012), "Variance estimation using refitted cross-validation in ultrahigh dimensional regression," Journal of the Royal Statistical Society, Series B, 74, 37-65.

Fan, J. and Li, R. (2001), "Variable selection via nonconcave penalized likelihood and its oracle properties," Journal of the American Statistical Association, 96, 1348-1360.

Fan, J. and Lv, J. (2008), "Sure independence screening for ultrahigh dimensional feature space (with discussion)," Journal of the Royal Statistical Society, Series B, 70, 849-911.

Fan, J. and Peng, H. (2004), "Nonconcave penalized likelihood with a diverging number of parameters," Ann. Statist., 32, 928-961.

Fan, J. and Song, R. (2010), "Sure independence screening in generalized linear model with NP-dimensionality," Annals of Statistics, 38, 3567-3604.

Fithian, W., Sun, D., and Taylor, J. (2014), “Optimal Inference After Model Selection,” ArXiv:1410.2597.

Friedman, J., Hastie, T., and Tibshirani, R. (2008), "Sparse inverse covariance estimation with the graphical lasso," Biostatistics, 9, 432-441.

Gaither, A., Porter, D., Yao, Y., Borawski, J., Yang, G., Donovan, J., Sage, D., Slisz, J., Tran, M., Straub, C., Ramsey, T., Iourgenko, V., Huang, A., Chen, Y., Schlegel, R., Labow, M., Fawell, S., Sellers, W., and Zawel, L. (2007), "A Smac mimetic rescue screen reveals roles for inhibitor of apoptosis proteins in tumor necrosis factor-alpha signaling," Cancer Research, 67, 11493-11498.

Grünwald, V. and Hidalgo, M. (2003), "Developing inhibitors of the epidermal growth factor receptor for cancer treatment," Journal of the National Cancer Institute, 95, 851-867.

Hadley, K. and Hendricks, D. (2014), "Use of NQO1 status as a selective biomarker for oesophageal squamous cell carcinomas with greater sensitivity to 17-AAG," BMC Cancer, 14, 1-8.

Hans, C., Dobra, A., and West, M. (2007), "Shotgun Stochastic Search for Regression With Many Candidate Predictors," Journal of the American Statistical Association, 102, 507-516.

Henze, N. and Zirkler, B. (1990), "A class of invariant consistent tests for multivariate normality," Communications in statistics - Theory and Methods, 10, 3595-3617. 
Holm, S. (1979), "A simple sequentially rejective multiple test procedure," Scandinavian Journal of Statistics, $6,65-70$.

Javanmard, A. and Montanari, A. (2014), "Confidence intervals and hypothesis testing for high-dimensional regression," Journal of Machine Learning Research, 15, 2869-2909.

Jia, B., Liang, F., Shi, R., and Xu, S. (2018), "equSA: Estimate Directed and Undirected Graphical Models and Construct Networks," $R$ package version 1.1.5, https://CRAN.R-project.org/package=equSA.

Lauritzen, S. (1996), Graphical Models, Oxford University Press.

Lawrence, R. and Salgia, R. (2010), "MET molecular mechanisms and therapiesin lung cancer," Cell Adhesion \& Migration, 4, 146-152.

Lee, H., Hanibuchi, M., Lim, S.-J., Yu, H., Kim, M., He, J., Langley, R., Lehembre, F., Regenass, U., and Fidler, I. (2016a), "Treatment of experimental human breast cancer and lung cancer brain metastases in mice by macitentan, a dual antagonist of endothelin receptors, combined with paclitaxel," NeuroOncology, $18,486-496$.

Lee, J. D. and Hastie, T. J. (2015), "Learning the structure of mixed graphical models," Journal of Computational and Graphical Statistics, 24, 230-253.

Lee, J. D., Sun, D. L., Sun, Y., and Taylor, J. E. (2016b), "Exact post-selection inference, with application to the lasso," Annals of Statistics, 44, 907-927.

Liang, F., Song, Q., and Qiu, P. (2015), "An Equivalent Measure of Partial Correlation Coefficients for High Dimensional Gaussian Graphical Models," Journal of the American Statistical Association, 110, 1248-1265.

Liang, F., Song, Q., and Yu, K. (2013), "Bayesian Subset Modeling for High Dimensional Generalized Linear Models," Journal of the American Statistical Association, 108, 589-606.

Liang, F. and Zhang, J. (2008), "Estimating FDR under general dependence using stochastic approximation," Biometrika, 95, 961-977.

Liu, H., Lafferty, J., and Wasserman, L. (2009), "The nonparanormal: Semiparametric estimation of high dimensional undirected graphs," J. Mach. Learn. Res., 10, 2295-2328.

Liu, Y. and Yu, B. (2013), "Asymptotic properties of Lasso+mLS and Lasso+Ridge in sparse high-dimensional linear regression," Electron. J. Stat., 7, 3124-3169.

Lockhart, R., Taylor, J., Tibshirani, R., and Tibshirani, B. (2014), "A significant test for the Lasso," Annals of Statistics, 42, 413-468.

Luo, S., Song, R., and Witten, D. (2014), "Sure Screening for Gaussian Graphical Models," ArXiv e-prints.

McCullagh, P. and Nelder, J. A. (1989), Generalized linear models (Second edition), London: Chapman \& Hall.

Meek, C. (1995), "Causal inference and causal explanation with background knowledge," in Proceedings of the 11th Conference on Uncertainty in Artificial Intelligence, pp. 403-410. 
Meier, L., Dezeure, R., Meinshausen, N., Maechler, M., and Buehlmann, P. (2016), "High-Dimensional Inference," https://cran.r-project.org/.

Meinshausen, N. (2007), "Relaxed Lasso," Computational Statistics \& Data Analysis, 52, 374-393.

- (2015), "Group bound: confidence intervals for groups of variables in sparse high dimensional regression without assumptions on the design," Journal of the Royal Statistical Society, Series B, 77, 923-945.

Meinshausen, N. and Bühlmann, P. (2006), "High-dimensional graphs and variable selection with the Lasso," Annals of Statistics, 34, 1436-1462.

Meinshausen, N. and Bühlmann, P. (2006), "High-dimensional graphs and variable selection with the Lasso," Ann. Statist., 34, 1436-1462.

Meinshausen, N., Meier, L., and Bühlmann, P. (2009), "p-values for high-dimensional regression," Journal of the American Statistical Association, 104, 1671-1681.

Moriwaki, K., Bertin, J., Gough, P., Orlowski, G., and Chan, F. (2015), "Differential roles of RIPK1 and RIPK3 in TNF-induced necroptosis and chemotherapeutic agent-induced cell death," Cell Death $\mathscr{E}$ Disease, 6, e1636.

Ooe, A., Kato, K., and Noguchi, S. (2007), "Possible involvement of CCT5, RGS3, and YKT6 genes upregulated in p53-mutated tumors in resistance to docetaxel in human breast cancers," Breast Cancer Research and Treatment, 101, 305-315.

Pellet, J.-P. and Elisseeff, A. (2008), "Using Markov blankets for causal structure learning," Journal of Machine Learning Research, 9, 1295-1342.

Portnoy, S. (1986), "On the central limit theorem in $R^{p}$ when $p \rightarrow \infty$," Probability Theory and Related Fields, $73,571-583$.

- (1989), "Asymptotic behavior of likelihood methods for exponential families when the number of parameters tend to infinity," Annals of Statistics, 16, 356-366.

Saldana, D. and Feng, Y. (2018), "SIS: An R package for Sure Independence Screening in Ultrahigh-dimensional Statistical Models," Journal of Statistical Softward, 83, 1-25.

Song, Q. and Liang, F. (2015a), "High Dimensional Variable Selection with Reciprocal L1-Regularization," Journal of the American Statistical Association, 110, 1607-1620.

- (2015b), "A Split-and-Merge Bayesian Variable Selection Approach for Ultra-high dimensional Regression," Journal of the Royal Statistical Society, Series B, 77, 947-972.

Spirtes, P., Glymour, C., and Scheines, R. (2000), Causation, Prediction, and Search, Boston: MIT Press.

Storey, J. (2002), "A direct approach to false discovery rates," Journal of the Royal Statistical Society, Series $B, 64,479-498$. 
Tibshirani, R. (1996), "Regression shrinkage and selection via the LASSO," Journal of the Royal Statistical Society, Series B, 58, 267-288.

Tibshirani, R. J., Taylor, J., Lockhart, R., and Tibshirani, R. (2016), "Exact Post-Selection Inference for Sequential Regression Procedures," Journal of the American Statistical Association, 111, 600-620.

van de Geer, S. and Bühlmann, P. (2009), "On the conditions used to prove oracle results for the Lasso," Electron. J. Stat., 3, 1360-1392.

van de Geer, S., Bühlmann, P., Ritov, Y., and Dezeure, R. (2014), "On asymptotically optimal confidence regions and tests for high-dimensional models," Ann. Statist., 42, 1166-1202.

Wasserman, L. and Roeder, K. (2009), "High-Dimensional variable selection," Annals of Statistics, 37, 21782201.

Witten, D., Friedman, J., and Simon, N. (2011), "New insights and faster computations for the graphical Lasso," Journal of Computational and Graphical Statistics, 20, 892-900.

Xu, S., Jia, B., and Liang, F. (2019), "Learning moral graphs in construction of high-dimensional Bayesian networks for mixed data," Neural Computation, 31, 1183-1214.

Xue, J. and Liang, F. (2017), "A Robust Model-Free Feature screening Method for Ultrahigh dimensional Data," Journal of Computational and Graphical Statistics, 26, 803-818.

Yang, Y. (2017), "Statistical inference for high dimensional regression via constrained Lasso," arXiv:1704.05098v1.

Yuan, M. and Lin, Y. (2007), "Model selection and estimation in the Gaussian graphical model," Biometrika, 95, 19-35.

Zhang, C.-H. (2010), "Nearly unbiased variable selection under minimax concave penalty," Annals of Statistics, 38, 894-942.

Zhang, C.-H. and Zhang, S. S. (2014), "Confidence intervals for low dimensional parameters in high dimensional linear models," Journal of the Royal Statistical Society: Series B (Statistical Methodology), 76, 217-242.

Zhang, X. and Cheng, G. (2017), "Simultaneous Inference for High-Dimensional Linear Models," Journal of the American Statistical Association, 112, 757-768.

Zoppoli, G., Regairaz, M., Leo, E., Reinhold, W., Varma, S., Ballestrero, A., Doroshow, J., and Pommier, Y. (2012), "Putative DNA/RNA helicase schlafenl1 (SLFN11) sensitizes cancer cells to DNA-damaging agents," Proceedings of the National Academy of Sciences USA, 109, 15030-15035.

Zou, H. (2006), "The adptive lasso and its oracle properties," Annals of Statistics, 38, 894-942. 